\title{
Lifetimes of near eternal false vacua
}

\author{
Aleksey Cherman ${ }^{*}$ and Theodore Jacobson ${ }^{\dagger}$ \\ School of Physics and Astronomy, University of Minnesota, Minneapolis, Minnesota 55455, USA
}

(Received 25 January 2021; accepted 16 April 2021; published 17 May 2021)

\begin{abstract}
We consider examples of long-lived false vacua in quantum field theory that arise from so-called 'universes'. These false vacua are protected by a $(d-1)$-form global symmetry, where $d$ is the dimension of spacetime. The lifetimes of the false vacua are set by UV data: the tension of $(d-2)$-branes charged under a $(d-2)$-form gauge symmetry. The lifetimes can be made parametrically long even when the difference in energy density between the false and true vacua is large compared to the natural scales of the field theory. We study examples of near-eternal false vacua in Abelian gauge theories in two dimensions and in four-dimensional QCD. In both cases, it is possible to view the $(d-1)$-form symmetries as arising from a modification of the sum over instantons. We find that the modification of the instanton sum in 4d QCD leads to a higher-group symmetry structure involving the 3-form and conventional 0-form global symmetries.
\end{abstract}

DOI: 10.1103/PhysRevD.103.105012

\section{INTRODUCTION}

False vacua are commonplace in quantum field theory (QFT). But we are used to the idea that if we wait long enough, the system always ends up in the true vacuum [1-6]. Indeed, suppose we prepare a system in a false vacuum state. In familiar situations, false vacuum decay proceeds by bubble nucleation: there is a nonzero probability for a bubble of the true vacuum to appear thanks to quantum or thermal fluctuations. Heuristically, small bubbles collapse to zero size due to the dominance of the bubble wall tension in their energy, but if a large enough bubble of the true vacuum appears, then the system can lower its energy by making the bubble bigger and bigger due to the difference in energy densities between the true and false vacua. As a result, sufficiently large bubbles expand and convert the entire system to the true vacuum. The lifetime of the false vacuum decreases as the difference in energy densities between the false and true vacuum is increased.

Taken at face value, the discussion above might suggest that the notion of "eternal false vacua" in the title of this paper is an oxymoron: a false vacuum should never be eternal in QFT. However, some recent theoretical developments imply that in fact eternal false vacua do exist in some

\footnotetext{
*acherman@umn.edu

jaco2585@umn.edu
}

Published by the American Physical Society under the terms of the Creative Commons Attribution 4.0 International license. Further distribution of this work must maintain attribution to the author(s) and the published article's title, journal citation, and DOI. Funded by SCOAP ${ }^{3}$.
QFTs. The examples we will study the following three properties ${ }^{1}$ :

(1) a $\mathbb{Z}_{p}(d-1)$-form global symmetry, denoted by $\mathbb{Z}_{p}^{(d-1)}$.

(2) some point in its parameter space where a global 0 -form symmetry $G^{(0)}$ appears.

(3) a mixed 't Hooft anomaly involving $G^{(0)}$ and $\mathbb{Z}_{p}^{(d-1)}$. The first (and consequently third) properties above may seem rather exotic, but we will see that there are some very simple examples of theories with these properties.

In our examples $G^{(0)}$ will be an Abelian group. At the point in parameter space where $G^{(0)}$ is a symmetry, the long-distance effective field theory cannot be trivial due to the 't Hooft anomaly, and in the examples we will discuss $G^{(0)}$ is spontaneously broken, so that there are $\left|G^{(0)}\right|$ degenerate vacua. However, another consequence of the 't Hooft anomaly is that the would-be domain walls connecting these vacua have infinite tension. So even when the spatial volume is finite, the degenerate vacua cannot mix, in sharp contrast to more familiar examples of QFTs with spontaneously broken symmetries. We will follow [7-9] and call distinct vacua with the peculiar property above "universes." Indeed, so long as $\mathbb{Z}_{p}^{(d-1)}$ is not explicitly broken, no local measurement within a given universe can tell us anything about the other universes-

\footnotetext{
${ }^{1}$ These properties are sufficient but not necessary for the existence of universes. For instance, in 2d Maxwell theory the existence of a $U(1)$ electric 1-form global symmetry alone gives rise to eternal false vacua characterized by expectation values of the local operator $\star F=\frac{1}{2} \epsilon^{\mu \nu} F_{\mu \nu}$, which is nothing but a constant electric field.
} 
including whether they even exist. Only the behavior of $(d-1)$-dimensional extended operators can probe the differences between the universes. More physically, if the $\mathbb{Z}_{p}^{(d-1)}$ global symmetry is slightly broken, then the "disconnected" universes all get connected into one universe. The fact that a given QFT gives rise to distinct universes in the limit that $\mathbb{Z}_{p}^{(d-1)}$ becomes exact has important implications even when $\mathbb{Z}_{p}^{(d-1)}$ is slightly broken so that there is only one universe, which we will explore here.

Let us first suppose $\mathbb{Z}_{p}^{(d-1)}$ is not broken explicitly. Then we can ask what happens if we explicitly break the global symmetry $G^{(0)}$ by dialing parameters in the QFT. Clearly the Poincaré-invariant local minima of the quantum effective potential become nondegenerate, leading to the appearance of a unique minimum-energy vacuum. Normally, this would imply that a locally-stable vacuum with a nonminimal value of the energy density - a false vacuumwould decay to the true vacuum in the thermodynamic equilibrium limit. But here this decay is impossible, and the lifetime of the false vacuum is infinite. This should be viewed as a remnant consequence of the 't Hooft anomaly between $G^{(0)}$ and $\mathbb{Z}_{p}^{(d-1)}$. Bubbles of any finite size simply cannot appear, because the surface tension of such a bubble is infinite. To get a finite false-vacuum lifetime, one must also explicitly break the $\mathbb{Z}_{p}^{(d-1)}$ symmetry. Breaking the $\mathbb{Z}_{p}^{(d-1)}$ symmetry entails coupling the system to dynamical $(d-2)$-branes: objects with a $(d-1)$-dimensional world volume with a tension $T_{d-2}$. The wall tension of a bubble of true vacuum is $\sim T_{d-2}$. As a result, the false vacuum lifetime is determined by the exponential of the ratio of the tension $T_{d-2}$ of these branes and the dimensionful scales of the original QFT.

QFTs with unbroken $n$-form global symmetries are believed to be inconsistent with quantum gravity constraints (such QFTs are termed to be "in the swampland"), see, e.g., Ref. [10]. So for phenomenological applications of these ideas, one should assume the $(d-2)$-branes that are sources for the $\mathbb{Z}_{p}^{(d-1)}$ symmetry have a finite tension. This tension can be extremely large $\left(T_{d-2} \sim M_{\text {Planck }}^{d-1}\right)$ compared to the energy scales which are native to our $d$ dimensional QFT, leading to extremely long-lived false vacua, without any need to tune the parameters controlling the low-energy physics.

In what follows we will do a warm up in quantum mechanics, and then discuss some simple examples of universes in QFTs in $d=2$ and $d=4$. In $d=2$ we will focus on variants of the venerable Schwinger model: that is, quantum electrodynamics in $d=2$. In this simple example, there are two ways to interpret the $(d-1)$-form symmetry. One way to obtain the 1-form symmetry is to assume the matter fields have charge $p$, and then the $\mathbb{Z}_{p}^{(d-1)}$ symmetry is just the 1-form 'center' symmetry. The other way to obtain the 1 -form symmetry is to have matter with charge 1 , but modify the instanton sum to only include instantons with topological charge divisible by $p$. In either case, $G^{(0)}$ is a discrete chiral symmetry. The 1 -form symmetry can be broken by adding massive matter fields with appropriate gauge charges. The $2 \mathrm{~d}$ analysis can be done very explicitly using $2 \mathrm{~d}$ bosonization techniques.

After that we make the jump to $d=4$, where we study $S U(N)$ QCD with $N_{f}$ fundamental fermions and a modified instanton sum, generalizing the analysis of [8]. In this variant of QCD the instanton sum is modified such that only instantons with topological charge divisible by a positive integer $p$ are allowed to contribute to the path integral. Then $G^{(0)}=\mathbb{Z}_{2 N_{f} p}^{(0)}$ is a discrete chiral symmetry, while $\mathbb{Z}_{p}^{(d-1)}$ is a 3 -form symmetry when $d=4$. We show that the 3-form symmetry and the 0 -form continuous symmetry groups of QCD do not form a direct product. Instead, they mix nontrivially so that the symmetry group of QCD with a modified instanton sum should be thought of as a 4-group. Breaking the higher-form symmetry requires coupling our variant of $4 \mathrm{~d} \mathrm{QCD}$ to dynamical 2-branes. The hierarchy between the scale set by the 2-brane tension $T_{2}$ and the QCD scale $\Lambda_{\mathrm{QCD}}$ implies that false vacua in the modified QCD theory are very long lived.

\section{UNIVERSES IN QUANTUM MECHANICS}

\section{A. Eternal false vacua are trivial in quantum mechanics}

In quantum mechanics, we are used to the idea that excited states cannot decay provided they are exact eigenstates of the Hamiltonian. If the Hamiltonian enjoys some global symmetry $G$, then there are no transitions between an excited state carrying global charge to a lower energy zero charge state such as the vacuum. In this sense, the notion of an eternal false vacuum is somewhat trivial in quantum mechanics.

For instance, consider a particle in a symmetric doublewell potential. The spectrum organizes itself into representations of parity, which acts as $x \rightarrow-x$. The ground state is the symmetric combination of nodeless wave functions localized at the two wells, and is invariant under parity. The first excited state is the antisymmetric combination, which is odd under parity. On top of each of these states is a tower of excitations, each being even or odd under parity. We may view each tower as being distinct universes, in the sense that they are protected by a selection rule: only parity odd operators can connect states between the two sectors. (Of course, the excited states within each tower cannot decay either, since they are energy eigenstates.)

Going back to generalities, suppose the system has a second global symmetry $G^{\prime}$ which has a mixed 't Hooft anomaly with $G$. As a consequence, the ground state cannot be unique. We can choose a basis such that the lowest energy states are eigenstates of $G$, but not $G^{\prime}$ (they form a 
$G^{\prime}$ multiplet). In this way, the symmetry $G^{\prime}$, together with the 't Hooft anomaly, ensure that the universes associated with $G$ are degenerate. If we break $G^{\prime}$ explicitly this degeneracy is lost, though the universes remain.

An example of the above situation is furnished by a free particle on a circle with a $\theta$-angle [11-13]. At $\theta=\pi$ there is a 't Hooft anomaly between the $U(1)$ shift symmetry and charge conjugation, which together comprise an $O(2)$ symmetry. This symmetry is projectively realized on the Hilbert space. The spectrum consists of states $|n\rangle$ labeled by their $U(1)$ charge. According to the discussion above, each state is its own universe. The two lowest-energy universes $|0\rangle$ and $|1\rangle$ are degenerate and are exchanged by charge conjugation. Once we break charge conjugation by moving away from $\theta=\pi$, one of these states becomes the true vacuum. However, the $U(1)$ symmetry prohibits the decay of the higher-energy states to this unique vacuum.

The discussion so far serves to show that in quantum mechanics, universes and eternal false vacua are overlycomplicated ways of thinking about completely standard concepts. However, it will be useful to consider an additional quantum-mechanical example in more detail. The following model exhibits many of the features of the quantum field theories discussed later in the paper.

\section{B. A quantum mechanics warmup}

Consider a quantum mechanical system consisting of three particles with coordinates are $x, y, z$ respectively on a circle of radius $2 \pi L$ with Euclidean-time Lagrangian

$\mathcal{L}=\frac{1}{2 g}\left(\dot{x}^{2}+\dot{y}^{2}\right)+\frac{i}{2 \pi} x \dot{y}+\frac{i}{2 \pi} \chi(\dot{y}-p \dot{z})+V(x, y, z)$,

where we took units where $L=1$ and $p \in \mathbb{Z}_{\geq 0}{ }^{2}$ We assume that the $x$ and $z$ periodicities in the potential are $2 \pi / p$ and the $y$ periodicity in the potential is $2 \pi$. Although the variables $\chi$ and $z$ do not have kinetic terms, they are dynamical in the sense that we integrate over all configurations of $\chi$ and $z$ in the path integral. The $2 \pi$-periodic variable $\chi$ plays the role of a Lagrange multiplier setting $\dot{y}=p \dot{z}$. Consequently, winding numbers of $y$ are constrained to be multiples of $p$,

$$
\int d y=p \int d z \in 2 \pi p \mathbb{Z}
$$

To keep things simple, in the following we will set $V(x, y, z)=V(x)$ below unless specified otherwise. This

\footnotetext{
${ }^{2}$ This model is related to the small-circle limit of the charge- $p$ Schwinger model on $\mathbb{R} \times S^{1}$ which appears in [14]. For the precise connection between two-dimensional Abelian theories with nonminimal charge and modified instanton sums, see Sec. III B.
}

allows us to demonstrate many features exactly (by integrating out $z$ explicitly, for instance) rather than making general statements and then checking them in perturbation theory.

The equation of motion for $z$ sets $\dot{\chi}=0$, and summing over winding configurations with $\int d z \in 2 \pi \mathbb{Z}$ sets $\chi=$ $2 \pi m / p$ for $m \in \mathbb{Z}$. The variable $e^{i \chi}$ is therefore $\mathbb{Z}_{p}$-valued, and summing over its discrete values enforces the constraint $\int d y \in 2 \pi p \mathbb{Z}$ as a delta function in the path integral. ${ }^{3}$

For our purposes the relevant symmetries of the model are the two discrete $\mathbb{Z}_{p}$ shift symmetries

$$
\begin{aligned}
& \left(\mathbb{Z}_{p}\right)_{x}: x \rightarrow x+\frac{2 \pi}{p}, \quad \chi \rightarrow \chi-\frac{2 \pi}{p}, \\
& \left(\mathbb{Z}_{p}\right)_{z}: z \rightarrow z+\frac{2 \pi}{p} .
\end{aligned}
$$

The symmetry operator of $\left(\mathbb{Z}_{p}\right)_{z}$ is $e^{-i \chi}$, in the sense that

$$
\left\langle e^{-i \chi\left(t_{0}\right)} e^{i z\left(t_{1}\right)}\right\rangle=e^{\frac{2 \pi i}{p} \Theta\left(t_{1}-t_{0}\right)}\left\langle e^{i z\left(t_{1}\right)}\right\rangle .
$$

To verify this relation one can write

$$
\left\langle e^{-i \chi\left(t_{0}\right)} e^{i z\left(t_{1}\right)}\right\rangle=\frac{1}{Z} \int \mathcal{D} x \mathcal{D} y \mathcal{D} z \mathcal{D} \chi e^{-(S+\delta S)} e^{i z\left(t_{1}\right)}
$$

where $\delta S=i \int d t \chi(t) \delta\left(t_{0}\right)$. We can cancel this shift in the action by a shift of $z(t) \rightarrow z(t)+\frac{2 \pi}{p} \Theta\left(t-t_{0}\right)$, which leads to

$$
\left\langle e^{-i \chi\left(t_{0}\right)} e^{i z\left(t_{1}\right)}\right\rangle=\frac{e^{i \frac{2 \pi}{p} \Theta\left(t_{1}-t_{0}\right)}}{Z} \int \mathcal{D} x \mathcal{D} y \mathcal{D} z \mathcal{D} \chi e^{-S} e^{i z\left(t_{1}\right)},
$$

reproducing (2.4). Since $e^{-i \chi}$ itself is charged under $\left(\mathbb{Z}_{p}\right)_{x}$, these two shift symmetries have a mixed 't Hooft anomaly [15]. The arguments summarized in the Introduction suggest that this ought to give rise to $p$ universes.

To see these distinct universes explicitly, it is convenient to integrate out $y$ and $z$ to isolate the dynamics of $x$. Since neither $y$ nor $z$ appear in the Lagrangian explicitly, we can instead integrate over $\dot{y}$ and $\dot{z}$ at the expense of constraining $\frac{1}{2 \pi} \int d y$ and $\frac{1}{2 \pi} \int d z$ to be integers. This can be achieved with the discrete delta functions

$$
\int \mathcal{D} y \mathcal{D} z \rightarrow \sum_{k \in \mathbb{Z}} \sum_{m \in \mathbb{Z}} \int \mathcal{D} \dot{y} \mathcal{D} \dot{z} e^{i k \int d t \dot{y}} e^{i m \int d t \dot{z}}
$$

Integrating out $\dot{y}$ and $\dot{z}$, we find

\footnotetext{
${ }^{3}$ This constraint on the winding numbers of $y$ still holds when $\chi$ and $z$ have kinetic terms, despite the fact that $\chi$ is no longer a discrete-valued field in this case. For simplicity we set these kinetic terms to zero.
} 


$$
Z=\sum_{k \in \mathbb{Z}} \sum_{m \in \mathbb{Z}} \int \mathcal{D} x \mathcal{D} z \mathcal{D} \xi e^{-\int d t \frac{1}{2 g} \dot{x}^{2}+V(x)+\frac{g}{8 \pi^{2}}(x+\xi-2 \pi k)^{2}-i \dot{z}\left(\frac{p}{2 \pi} \xi+m\right)}
$$

$$
\begin{aligned}
& \sim \sum_{m=0}^{p-1} \sum_{k \in \mathbb{Z}} \int d t \mathcal{D} x e^{-\int \frac{1}{2 g} \dot{x}^{2}+V(x)+\frac{g}{8 \pi^{2}}\left(x-\frac{2 \pi m}{p}-2 \pi k\right)^{2}} \\
& \equiv \sum_{m=0}^{p-1} Z_{m}
\end{aligned}
$$

Upon integrating out the auxiliary fields the partition function becomes highly nonlocal, decomposing into a sum of partition functions $Z_{m}$ labeled by the corresponding value of the Lagrange multiplier $\chi=2 \pi m / p$. The $p$ different terms on the right-hand side of (2.9) are the universes we advertised earlier.

On the other hand, if we restrict our attention to constant values of $x$ and take the Euclidean time direction to be large, then the effect of integrating out $y, z, \chi$ is to produce a local effective potential,

$$
V_{\mathrm{eff}}(x)=V(x)+\min _{k \in \mathbb{Z}} \frac{g}{8 \pi^{2}}\left(x-\frac{2 \pi k}{p}\right)^{2},
$$

with degenerate minima related by $\left(\mathbb{Z}_{p}\right)_{x}$. On the one hand, these $p$ vacua appear to have matched the ' $t$ Hooft anomaly between $\left(\mathbb{Z}_{p}\right)_{x}$ and $\left(\mathbb{Z}_{p}\right)_{z}$. At the same time, the naive quantum-mechanical expectation is that the true ground state of the system is described by the $\left(\mathbb{Z}_{p}\right)_{x}$-symmetric linear combination of wave functions localized at each minimum. Put differently, one might expect instantons to lift the $p$-fold degeneracy by a nonperturbative amount. However, there are no instanton configurations connecting the minima of the potential (2.10). To consider dynamical configurations we must pass to the nonlocal expression (2.9), where it becomes obvious that "nearest-neighbor instantons" are not sensible field configurations because they would have to connect different terms in the path integral decomposition (i.e., universes).

An immediate corollary is that the $\left(\mathbb{Z}_{p}\right)_{x}$ symmetry is spontaneously broken, contrary to the usual lore prohibiting such a conclusion in quantum mechanics. To see this, let us evaluate the expectation value $\left\langle e^{i x}\right\rangle$ with a small $\left(\mathbb{Z}_{p}\right)_{x}$-breaking perturbation added to the action, and with time having finite extent $T$,

$$
\begin{aligned}
\left\langle e^{i x}\right\rangle & =\frac{1}{Z} \sum_{m=0}^{p-1} \sum_{k \in \mathbb{Z}} \int \mathcal{D} x e^{i x} e^{-\int d t \frac{1}{2 g} \dot{x}^{2}+V(x)+\frac{g}{8 \pi^{2}}\left(x-\frac{2 \pi m}{p}-2 \pi k\right)^{2}-\epsilon \cos (x)} \\
& =\frac{\sum_{m=0}^{p-1}\left\langle e^{i x}\right\rangle_{m} Z_{m}(\epsilon)}{\sum_{m=0}^{p-1} Z_{m}(\epsilon)}=\frac{\sum_{m=0}^{p-1}\left\langle e^{i x}\right\rangle_{m} e^{-T \mathcal{F}_{m}(\epsilon)}}{\sum_{m=0}^{p-1} e^{-T \mathcal{F}_{m}(\epsilon)}}
\end{aligned}
$$

where $\left\langle e^{i x}\right\rangle_{m}$ is the expectation value in the $m$ th universe with free energy $\mathcal{F}_{m}(\epsilon)$. When $\epsilon>0$, the free energy $\mathcal{F}_{0}(\epsilon)<\mathcal{F}_{i}(\epsilon)$ for $i>0$. Taking the limit $T \rightarrow \infty$, only a single term in (2.12) survives,

$$
\lim _{T \rightarrow \infty}\left\langle e^{i x}\right\rangle=\left\langle e^{i x}\right\rangle_{0}
$$

Finally, taking the symmetry-breaking parameter to zero, we find

$$
\lim _{\epsilon \rightarrow 0} \lim _{T \rightarrow \infty}\left\langle e^{i x}\right\rangle=\lim _{\epsilon \rightarrow 0}\left\langle e^{i x}\right\rangle_{0}=1,
$$

so that the $\left(\mathbb{Z}_{p}\right)_{x}$ symmetry is spontaneously broken. Note that in coming to this conclusion, the exact decomposition of the path integral into universes was crucial-without it, finite-action instantons would proliferate even in the $T \rightarrow$ $\infty$ limit, leading to a unique symmetric ground state. Finally, we note that the $p$ degenerate ground states match the 't Hooft anomaly between the two $\mathbb{Z}_{p}$ symmetries.

We can also see how these degenerate ground states appear from a Hamiltonian perspective. In Minkowski space, the Hamiltonian resulting from the Lagrangian (2.1) is

$$
\hat{H}=\frac{g}{2}\left[\hat{p}_{x}^{2}+\left(\hat{p}_{y}+\frac{\hat{p}_{z}}{p}-\frac{\hat{x}}{2 \pi}\right)^{2}\right]+V(\hat{x}, \hat{y}, \hat{z}) .
$$

In the Hamiltonian formalism the $\left(\mathbb{Z}_{p}\right)_{x}$ and $\left(\mathbb{Z}_{p}\right)_{z}$ shift symmetries are generated by the operators $\hat{U}_{x}=e^{i \frac{2 \pi}{p} \hat{p}_{x}} e^{-i \hat{z}}$ and $\hat{U}_{z}=e^{i \frac{2 \pi}{p} \hat{p}_{z}}$, respectively. Let $V(\hat{x}, \hat{y}, \hat{z})=0$ for simplicity. Then the above Hamiltonian is equivalent to a Landau problem on the torus with $p$ units of magnetic flux [14]. The system has $p$ degenerate ground states $|m\rangle$ on the torus identified by their eigenvalues $\hat{U}_{z}|m\rangle=e^{2 \pi i m / p}|m\rangle$ under $\left(\mathbb{Z}_{p}\right)_{z}$. The wave functions are

$\Psi_{m}(\vec{x})=\langle\vec{x} \mid m\rangle=\frac{1}{2 \pi}\left(\frac{1}{2 \pi^{2}}\right)^{1 / 4} \sum_{k \in \mathbb{Z}} e^{i k y} e^{i m z} e^{-\frac{1}{4 \pi}\left(x-2 \pi k-\frac{2 \pi m}{p}\right)^{2}}$.

The $p$ ground states have energy $E=g / 4 \pi$ and are permuted by $\left(\mathbb{Z}_{p}\right)_{x}$ as $\hat{U}_{x}|m\rangle=|m-1\rangle$. The symmetry operators therefore satisfy the relation $\hat{U}_{x} \hat{U}_{z}=e^{2 \pi i / p} \hat{U}_{z} \hat{U}_{x}$ corresponding to a central extension of $\mathbb{Z}_{p} \times \mathbb{Z}_{p}$ to the WeylHeisenberg group. This is the manifestation of the mixed anomaly.

It is important to note that the existence of $p$ universes is protected by the $\left(\mathbb{Z}_{p}\right)_{z}$ symmetry alone, and does not rely on the existence of the $\left(\mathbb{Z}_{p}\right)_{x}$ symmetry nor the 't Hooft anomaly between them. To see this, we can again add the $\left(\mathbb{Z}_{p}\right)_{x}$-breaking term $\epsilon \cos (x)$ to the Lagrangian. As shown in Fig. 1(b), such a term lifts the degeneracy between universes, but the universes remain. Despite the fact that we 


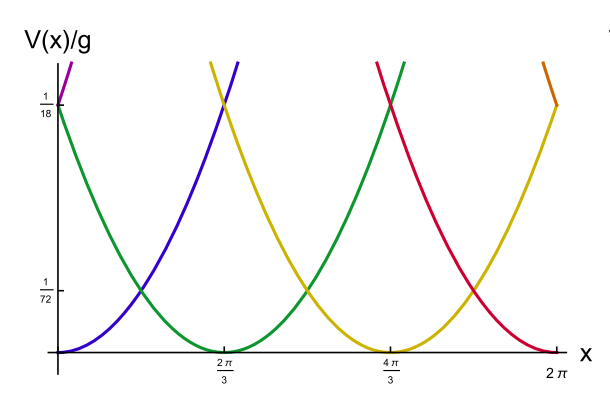

(a) $\mu=0, \epsilon=0$

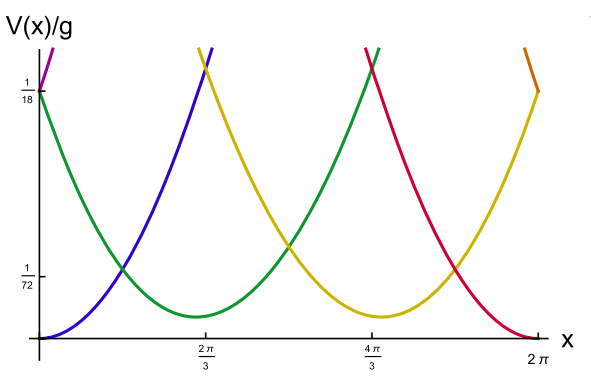

(b) $\mu=0, \epsilon \neq 0$

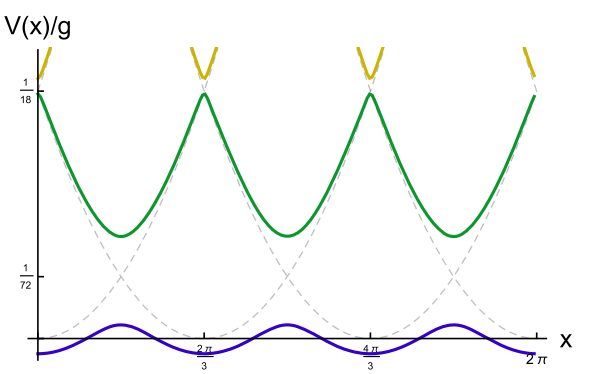

(c) $\mu \neq 0, \epsilon=0$

FIG. 1. Effective potential $V_{\text {eff }}(x)$ for $p=3$, for different realizations of the $\left(\mathbb{Z}_{p}\right)_{x}$ and $\left(\mathbb{Z}_{p}\right)_{z}$ symmetries. The potential on the right is obtained by numerically diagonalizing the Hamiltonian (2.17) with $\mu=.02 g$. The curvature of the potential near $x=0$ is roughly $m_{\text {eff }} \approx .02 g<\sqrt{p} \mu$, so the Born-Oppenheimer approximation is justified.

can make the difference in energy densities between neighboring vacua large by increasing $\epsilon$, if we prepare the system in one of the false vacuum states it cannot tunnel into the true vacuum. Hence, the false vacua are eternal.

On the other hand, we can leave $\left(\mathbb{Z}_{p}\right)_{x}$ intact and break the $\left(\mathbb{Z}_{p}\right)_{z}$ symmetry explicitly by (for example) including a $V(\hat{x}, \hat{y}, \hat{z})=\cos (\hat{z})$ potential,

$$
\hat{H}=\frac{g}{2}\left[\hat{p}_{x}^{2}+\left(\hat{p}_{y}+\frac{\hat{p}_{z}}{p}-\frac{\hat{x}}{2 \pi}\right)^{2}\right]+\mu \cos (\hat{z})
$$

This leaves a single universe with degenerate vacua related by the remaining $\left(\mathbb{Z}_{p}\right)_{x}$ symmetry. Since these vacua now exist in the same universe, finite-action tunneling events will proliferate and give rise to a unique symmetric vacuum. In fact, the ground state remains unique even as we take $\mu \rightarrow 0$. On the other hand, we know that when $\mu$ is strictly vanishing there are $p$ degenerate ground states.

To see this explicitly we can treat the variable $x$ within the Born-Oppenheimer approximation. Integrating out the 'fast' mode $z$ gives an effective potential for $x$ which is the analog of Eq. (2.10) when the $\left(\mathbb{Z}_{p}\right)_{z}$ symmetry is broken. The Born-Oppenheimer approximation is justified as long as the curvature of the induced effective potential is smaller than $\sqrt{p} \mu$, which can easily be arranged. Though the $\left(\mathbb{Z}_{p}\right)_{z}$-breaking potential is nonlinear, this process can be done numerically. As shown in Fig. 1(c), the level-crossings are avoided and the spectrum organizes itself into bands. Within the lowest band, instantons proliferate and give rise to a unique ground state. We can even compute the instanton action for nearest-neighbor tunneling in the limit $\mu \rightarrow 0$. In this limit the effective potential reduces to (2.10). When $V(x)=0$, the instanton action is $S_{I}=\frac{\pi}{2 p^{2}}$, which clearly shows no singularity as $\mu \rightarrow 0$. Again, this signals a non-uniformity in the symmetry-restoring limit: we know from our above arguments that $\mu=0$ is a distinguished point in parameter space where tunneling is completely suppressed, i.e., where $S_{I}$ is infinite.

\section{UNIVERSES IN THE MASSLESS SCHWINGER MODEL AND ITS DEFORMATIONS}

We now move on to examples of universes and eternal false vacua in quantum field theory. It is crucial to note that while in quantum mechanics an eternal false vacuum is a trivial concept, it becomes nontrivial in relativistic QFT, where vacua should be Poincaré-invariant. For instance, while an ordinary 0 -form global symmetry may ensure that certain single-particle states are stable, such states are not eternal false vacua as they are not Poincaré-invariant. Indeed, eternal false vacua can only exist due to $(d-1)$-form symmetries, which are higherform symmetries in $d \geq 2$.

In this section we analyze variations of the Schwinger model: two-dimensional $U(1)$ gauge theory with a charged Dirac fermion. We begin by taking the fermion to be massless, with charge $p \in \mathbb{Z}$, with $|p|>1$. Many aspects of the Schwinger model with nonminimal charge have been explored in recent works [9,14,16-19], where the mixed anomaly between the 0 -form chiral and 1-form center symmetries plays a central role. Reference [17] identified the $p$ vacuum branches of the theory as belonging to different universes (this was also discussed more recently in [9] using the language of universes), and explained how (de)confinement can be understood in terms of the energy densities in each universe. While our discussion in this section contains some overlap with Refs. [9,17], our main goal is to provide a self-contained discussion of how universes arise in the model, and in particular how eternal false vacua appear when the fermion is given a finite mass. We give parametric estimates of the lifetimes of false vacua when both chiral and center symmetry-breaking deformations are included. A discussion of false vacua similar in spirit to our analysis appears in Ref. [20] which, motivated by axion monodromy models of inflation, examines twodimensional theories featuring multibranched potentials with metastable vacua.

In Sec. III B, we show how the charge- $p$ Schwinger model can be understood as a charge-1 Schwinger model with a modified instanton sum. This perspective allows us 
to generalize our discussion of universes and eternal false vacua in two-dimensions to higher dimensions. We discuss the symmetries and $\theta$-dependence of the modified model in detail.

\section{A. Charge- $p$ Schwinger model}

The Euclidean Lagrangian of the massless charge- $p$ Schwinger model is

$\mathcal{L}=\frac{1}{4 e^{2}} f_{\mu \nu} f^{\mu \nu}+\frac{i \theta}{2 \pi} \epsilon^{\mu \nu} \partial_{\mu} a_{\nu}+\bar{\psi} \gamma^{\mu}\left(\partial_{\mu}-i p a_{\mu}\right) \psi$,

where $f_{\mu \nu}=\partial_{\mu} a_{\nu}-\partial_{\nu} a_{\mu}$. We assume that the theory is defined on a closed 2-manifold so that the topological charges are quantized as

$$
\int \epsilon^{\mu \nu} \partial_{\mu} a_{\nu}=\int d a \in 2 \pi \mathbb{Z} .
$$

The theory has a $\mathbb{Z}_{2 p}^{(0)} 0$-form chiral symmetry, but the $\mathbb{Z}_{2}$ subgroup generated by $(-1)^{F}$ is actually non-chiral and lies within the $U(1)$ gauge group. We will focus on the faithfully acting $\mathbb{Z}_{p}^{(0)}=\mathbb{Z}_{2 p}^{(0)} / \mathbb{Z}_{2}$ chiral symmetry below. The theory also has a 1 -form $\mathbb{Z}_{p}^{(1)}$ center symmetry. The bosonized form of the massless charge- $p$ Schwinger model is $[21,22]$

$$
\mathcal{L}=\frac{1}{2 e^{2}}|d a|^{2}+\frac{1}{8 \pi}|d \varphi|^{2}+\frac{i p}{2 \pi} \varphi \wedge d a+\frac{i \theta}{2 \pi} d a,
$$

where we have switched to form notation, so that $a=$ $a_{\mu} d x^{\mu}$ and used the shorthand $|\omega|^{2}=\omega \wedge \star \omega$. Here $\varphi$ is a $2 \pi$-periodic scalar and the discrete chiral symmetry becomes the shift symmetry $\varphi \rightarrow \varphi+2 \pi / p$. The 1 -form $\mathbb{Z}_{p}^{(1)}$ symmetry acts by shifting $a \rightarrow a+\lambda$ by a flat connection with $\int \lambda=2 \pi \mathbb{Z} / p$. The $\mathbb{Z}_{p}^{(0)}$ and $\mathbb{Z}_{p}^{(1)}$ symmetries have a mixed anomaly $[16,18,19]$, which can be seen by turning on an appropriate background 2-form gauge field for the 1-form symmetry.

An important point is that the symmetry operator of the 1-form symmetry is a local operator, which up to a normalization constant is given by $U_{m}(x)=$ $e^{i \frac{2 \pi m}{p}\left(-\frac{i}{e^{2}} \star d a+\frac{p}{2 \pi} \varphi\right)}$. Indeed, the equation of motion for the gauge field can be written as

$$
d\left(-\frac{i}{e^{2}} \star d a+\frac{p}{2 \pi} \varphi\right)=0 .
$$

The 0-form expression in parentheses is therefore a conserved quantity, and this can be used to show that $U_{m}(x)$ is a well-defined local topological operator so long as $m \in \mathbb{Z}$. The symmetry operator satisfies

$$
\left\langle U_{m}(x) e^{i \int_{C} a}\right\rangle=e^{\frac{2 \pi i m}{p} \ell(C, x)}\left\langle e^{i \int_{C} a}\right\rangle,
$$

where the linking number $\ell(C, x)=0$ or 1 depending on if the point $x$ is inside the closed contour $C$, which we take to be oriented and without self-intersections. So long as the 1 -form symmetry is not explicitly broken, correlation functions of $U_{m}(x)$ should be topological-which in the case of local operators, means independent of the position $x$. We will see that this enforces strict constraints on the possible dynamical domain wall configurations in which $\varphi$ approaches distinct values at asymptotic infinity. In particular, domain wall configurations where $U_{m}(x)$ depends on spacetime necessarily have infinite tension. While one can consider such domain walls as probes of the theory, they are not dynamical excitations [9].

In the deep IR limit $e^{2} \rightarrow \infty$, integrating out $a$ locally sets $d \varphi=0$, while the sum over topologically nontrivial sectors (global fluxes $\int d a \in 2 \pi \mathbb{Z}$ ) results in the constraint $\varphi=2 \pi \ell / p$, with $\ell \in \mathbb{Z}$. The field $\varphi$ is frozen to one of $p$ values, and there are no domain walls which interpolate between them. In this limit the distinct values $\ell \in$ $\{0, \ldots, p-1\}$ label distinct universes. Even away from the extreme IR limit the theory (3.3) is Gaussian and we can integrate out the gauge field by treating $F=d a$ as the fundamental degree of freedom. Imposing $U(1)$ flux quantization (read: the Bianchi identity) as a delta function constraint,

$$
\sum_{k \in \mathbb{Z}} e^{i k \int F}=\sum_{\nu \in \mathbb{Z}} \delta\left(\nu-\frac{1}{2 \pi} \int F\right),
$$

the path integral can be expressed as

$$
\begin{aligned}
Z(\theta)= & \int \mathcal{D} \varphi \sum_{k \in \mathbb{Z}} \exp \left\{-\int \frac{1}{8 \pi}|d \varphi|^{2}\right. \\
& \left.+\frac{1}{2}\left(\frac{e p}{2 \pi}\right)^{2}\left(\varphi+\frac{\theta}{p}-\frac{2 \pi k}{p}\right)^{2}\right\} .
\end{aligned}
$$

There is no longer a local effective Lagrangian for $\varphi$ : the partition function decomposes into a sum of distinct path integrals labeled by the integer $k .{ }^{4}$ The $p$ inequivalent terms in the above decomposition are distinct universes, labeled by the expectation value of the 1-form symmetry operator $\left\langle U_{1}(x)\right\rangle=e^{2 \pi i k / p}$.

In the infinite volume limit, the potential for the zero mode of $\varphi$, which we denote $\varphi_{0}$, can be written in a manifestly local way as

\footnotetext{
${ }^{4}$ If we decompose $k=p n+\ell$, where $n \in \mathbb{Z}$ and $\ell \in\{0, \ldots, p-1\}$, then $\ell$ can be interpreted as a discrete theta parameter of the charge- $p$ Schwinger model with the $\mathbb{Z}_{p}^{(1)} 1$-form symmetry gauged. At a technical level, minimizing over $n$ ensures that the potential is $2 \pi$-periodic with respect to $\varphi$ while the minimization over $\ell$ ensures the potential is $2 \pi$-periodic with respect to $\theta$.
} 


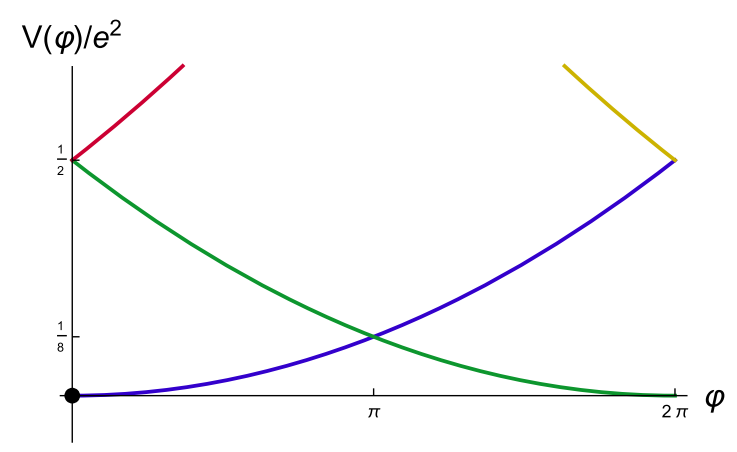

(a) $p=1$

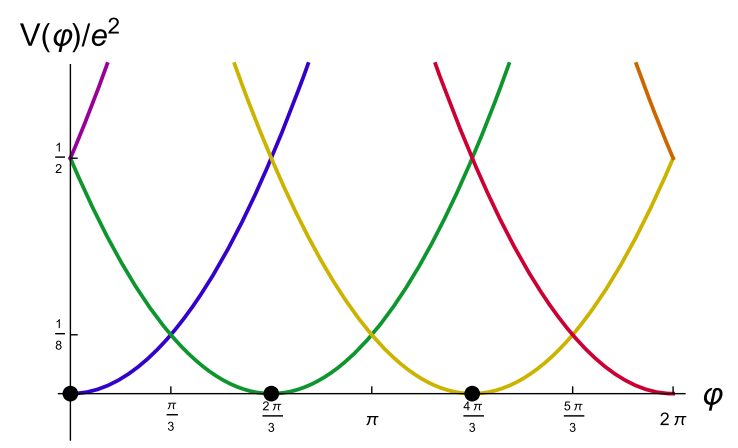

(b) $p=3$

FIG. 2. Multibranched structure of the effective potential for massless Schwinger models at $\theta=0$ with charge $p=1$ and $p=3$. For $p=1$, all branches correspond to the same universe. For $p=3$, there are three distinct universes.

$$
V\left(\varphi_{0}\right)=\min _{k \in \mathbb{Z}} \frac{1}{2}\left(\frac{e p}{2 \pi}\right)^{2}\left(\varphi_{0}+\frac{\theta}{p}-\frac{2 \pi k}{p}\right)^{2} .
$$

The potential in Eq. (3.8) has $p$ degenerate minima corresponding to $\varphi_{0}=(2 \pi k-\theta) / p$, which are illustrated in Fig. 2. These minima are related by the $\mathbb{Z}_{p}^{(0)}$ discrete shift symmetry, which is spontaneously broken. We emphasize that each chiral vacuum lies in a distinct universe. Hence, dynamical domain walls between neighboring vacua do not exist as they violate the "topological" property of the operator $U_{m}(x)$ (which is protected by the absence of charge- 1 matter and the resulting 1 -form $\mathbb{Z}_{p}^{(1)}$ symmetry). As a result, these states do not mix even when spacetime is compact: they belong to different universes. At the technical level, domain walls cannot exist because each $\mathbb{Z}_{p}^{(0)}$-breaking vacuum lies within a distinct branch of the effective potential.

At the risk of being pedantic, let us explain how spontaneous symmetry breaking occurs in the presence of universes. To conclude that a (0-form) symmetry is spontaneously broken one works in a large but finite spatial volume $V$ and introduces a symmetry-breaking perturbation at some scale $m$ which is small compared to the intrinsic scales (inverse correlation length) of the problem. Normally, we say that a symmetry is spontaneously broken when

$$
\lim _{m \rightarrow 0} \lim _{V \rightarrow \infty}\langle\mathcal{O}\rangle \neq 0
$$

for some local operator $\mathcal{O}$ which transforms under the symmetry. Importantly, the limits above do not commute, and it is always true that $\lim _{V \rightarrow \infty} \lim _{m \rightarrow 0}\langle\mathcal{O}\rangle=0$.

In the context of the Schwinger model, $m$ is a small chiral symmetry-breaking mass for the fermion, with the $\theta$ parameter absorbed into the phase of the fermion mass by a chiral rotation, $\arg (m)=\theta / p$. Using Eq. (3.7) we can write the expectation value of $e^{i \varphi} \sim \bar{\psi}_{L} \psi_{R}$ as

$$
\begin{aligned}
\left\langle e^{i \varphi}\right\rangle_{m=0} & =\lim _{\substack{m \rightarrow 0, \arg m \text { fixed }}} \lim _{L \rightarrow \infty} \frac{\sum_{k=0}^{p-1} \int \mathcal{D} \varphi e^{i \varphi} e^{-S_{k}(m)}}{\sum_{k=0}^{p-1} \int \mathcal{D} \varphi e^{-S_{k}(m)}} \\
& =\lim _{\substack{m \rightarrow 0, \arg m \text { fixed }}} \lim _{L \rightarrow \infty} \frac{\sum_{k=0}^{p-1}\left\langle e^{i \varphi}\right\rangle_{k} Z_{k}(m)}{\sum_{k=0}^{p-1} Z_{k}(m)}
\end{aligned}
$$

where $\left\langle e^{i \varphi}\right\rangle_{k}$ is the expectation value in the universe with action $S_{k}(m)$ and $L$ is the spatial volume. It is useful to trade $Z_{k}$ for the free energy $\mathcal{F}_{k}$ via $Z_{k}(m)=e^{-L T \mathcal{F}_{k}(m)}$, where $L T$ is the spacetime volume. For any given fixed $|m|$ and a generic value $\arg (m)$ the set $\left\{\mathcal{F}_{k}(m)\right\}$ has a unique minimum $\mathcal{F}_{k_{\min }}(m)$, but of course $k_{\min }$ depends on $\arg m .^{5}$ As a result, in the infinite volume limit only a single term in the sum contributes to the expectation value,

$$
\begin{aligned}
\left\langle e^{i \varphi}\right\rangle_{m=0} & =\lim _{\substack{m \rightarrow 0, \arg m \text { fixed }}} \lim _{L \rightarrow \infty} \frac{\sum_{k=0}^{p-1}\left\langle e^{i \varphi}\right\rangle_{k} e^{-L T \mathcal{F}_{k}(m)}}{\sum_{k=0}^{p-1} e^{-L T \mathcal{F}_{k}(m)}} \\
& =\lim _{\substack{m \rightarrow 0, \arg m \text { fixed }}}\left\langle e^{i \varphi}\right\rangle_{0}=e^{2 \pi i n / p},
\end{aligned}
$$

where the value of the integer $n$ depends on $\arg m=\theta / p$. If instead we had taken $m \rightarrow 0$ before $L \rightarrow \infty$, each universe would contribute equally to the expectation value, and we would erroneously conclude that $\left\langle e^{i \varphi}\right\rangle=$ $\sum_{k=0}^{p-1} e^{2 \pi i k / p}=0$.

We have seen that the $\mathbb{Z}_{p}^{(0)}$ chiral symmetry is spontaneously broken in the massless Schwinger model. When the fermion is massless the $\mathbb{Z}_{p}^{(1)}$ symmetry is also spontaneously broken. This is easily seen by noticing that the insertion of a charge- $q$ Wilson loop in the path integral shifts the theta angle by $\theta \rightarrow \theta-2 \pi q$ inside the loop. Hence, inside the loop, the partition function is that of the universe with an unshifted $\theta$ but with $k \rightarrow k+q$ relative to the outside. Since the universes are degenerate,

\footnotetext{
${ }^{5}$ When $\theta=\pi$ the minimum of $\left\{\mathcal{F}_{k}(m)\right\}$ becomes doubly degenerate, corresponding to the spontaneous breaking of charge conjugation. We will not consider this special case here.
} 


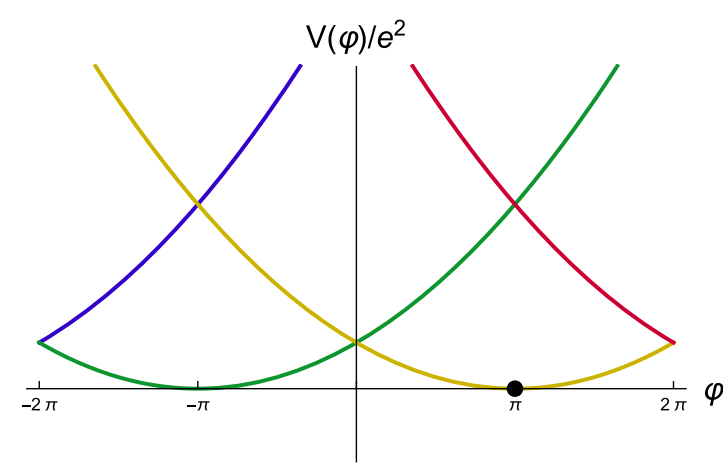

(a) $m=0$

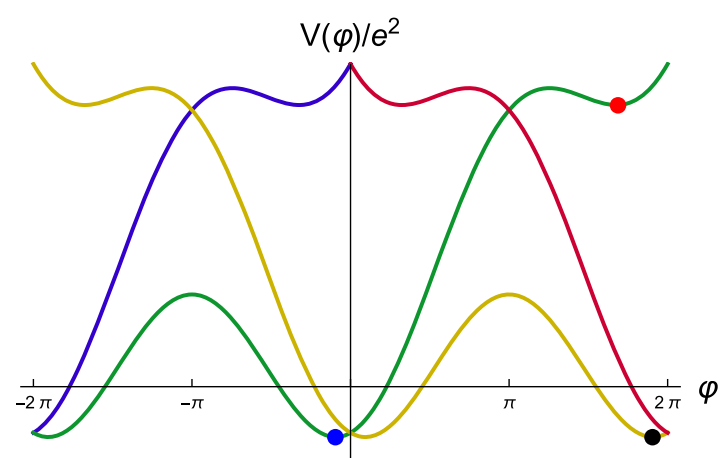

(b) $m>0$

FIG. 3. Multibranched structure of the effective potential for $p=1, \theta=\pi$. The metastable vacuum (red dot) in the $k=0$ branch unstable. It decays to the minimum marked by the blue dot (on the same $k=0$ branch). By a shift of $2 \pi$, this is equivalent to one of the true global minima, marked by the black dot.

the difference in energy density inside and outside the Wilson loop vanishes. As a result there is no area-law contribution to the Wilson loop expectation value, and $\mathbb{Z}_{p}^{(1)}$ is spontaneously broken. When the fermion mass $m \neq 0$ the theory becomes confining, with string tensions scaling like $m e$ or $m^{2}$ depending on whether $|m| \ll e$ or $|m| \gg e$ [9]. Of course, when we add charge- 1 matter to the theory the 1 -form symmetry is explicitly broken. In the following, we examine both symmetry-breaking perturbations of the massless Schwinger model in more detail.

Let us explicitly introduce a mass for the fermion, so that the bosonized potential is

$V\left(\varphi_{0}\right)=\min _{k \in \mathbb{Z}} \frac{1}{2}\left(\frac{e p}{2 \pi}\right)^{2}\left(\varphi_{0}+\frac{\theta}{p}-\frac{2 \pi k}{p}\right)^{2}-m \mu \cos \varphi_{0}$,

where $\mu$ is some scale determined by matching to the original fermionic theory. The mass term breaks the $\mathbb{Z}_{p}^{(0)}$ symmetry completely, and for generic values of $m, \theta$ there is a unique vacuum. The exception is at $\theta=\pi$, where two ground states emerge as one increases the mass. As an example, consider the $p=1$ case at $\theta=\pi$,

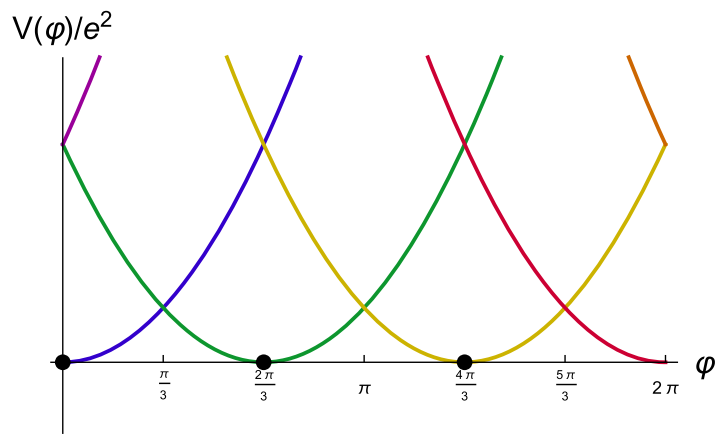

(a) $m=0$
$V\left(\varphi_{0}\right)=\min _{k \in \mathbb{Z}} \frac{1}{2}\left(\frac{e}{2 \pi}\right)^{2}\left(\varphi_{0}+\pi-2 \pi k\right)^{2}-m \mu \cos \varphi_{0}$.

For $m \mu \ll e^{2}$ the potential has a single minimum in the fundamental domain $\varphi_{0} \in(0,2 \pi)$ at $\varphi_{0}=\pi$. This minimum lies on the branch labeled by $k=1$. As we increase $m$ past a critical value, the potential develops two ground states related by charge conjugation. As we increase $m$ further, there is another critical value where local minima appear in the $k=0$ and $k=2$ branches, see Fig. 3. These metastable vacua can decay to one of the true vacua via a tunneling process. All of the branches can be connected via similar tunneling processes-this is possible because they lie within the same universe.

Let us contrast this to what happens when $p>1$. In Fig. 4 we compare the $p=3$ effective potential at $\theta=0$ for zero and nonzero mass. When $m=0$ there are three degenerate ground states which spontaneously break $\mathbb{Z}_{3}^{(0)}$. Each degenerate vacuum lies in a distinct universe. As soon as the fermion mass is nonzero the degeneracy is lifted and there is a unique vacuum which lies in the

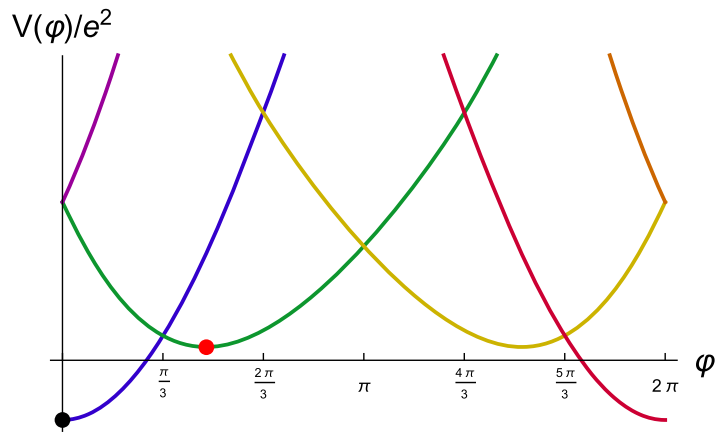

(b) $m>0$

FIG. 4. Multibranched structure of the effective potential for $p=3, \theta=0$. 


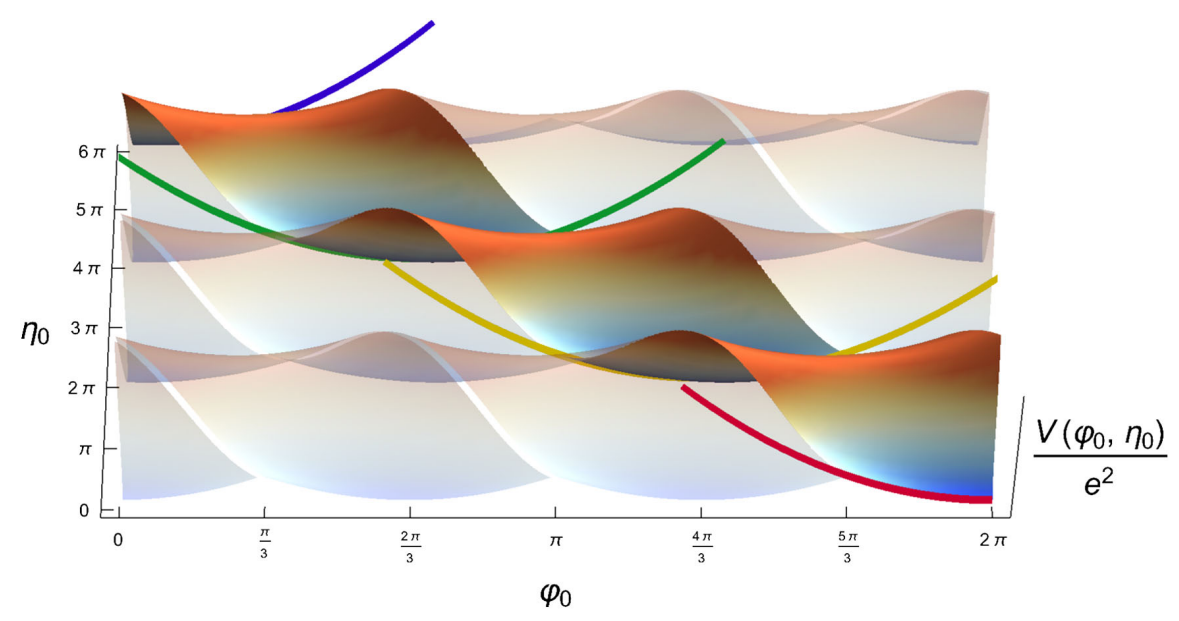

FIG. 5. Effective potential for bosonized scalars $\varphi, \eta$ with $p=3, m=0, M>0$. The four curves coincide with the four branches in Fig. 4.

branch labeled by $k=0$ mod 3. False vacua in different branches cannot decay to the true vacuum and are infinitely long-lived.

The infinite lifetimes of false vacua in the above examples with $p>1$ were protected by the 1 -form $\mathbb{Z}_{p}^{(1)}$ symmetry. This symmetry is explicitly broken when we introduce matter with minimal charge. To this end, let us introduce a heavy charge- 1 fermion $\zeta$ with mass $M$, with $M^{2} \gg e^{2}$. When $p$ is even, the $\zeta$ fermion number $(-1)^{F_{\zeta}}$ coincides with a gauge transformation, while when $p$ is odd, the overall fermion number $(-1)^{F}$ lies within the gauge group. Applying Abelian bosonization to $\psi \leftrightarrow \varphi$ and $\zeta \leftrightarrow \eta$ [22], we obtain a description of the theory with the overall fermion number gauged,

$$
\begin{aligned}
\mathcal{L}= & \frac{1}{2 e^{2}}|d a|^{2}+\frac{1}{8 \pi}|d \varphi|^{2}+\frac{1}{8 \pi}|d \eta|^{2} \\
& +\frac{i p}{2 \pi}\left(\varphi+\frac{\eta+\theta}{p}\right) \wedge d a-c M^{2} \cos \eta
\end{aligned}
$$

where $c$ is an $\mathcal{O}(1)$ constant. Again, we can integrate out the gauge field to obtain the effective potential for the zero-modes of $\varphi$ and $\eta$. This is shown in Fig. 5 for $p=3$. The bosonized charge- 1 fermion provides an extra direction in field space, and the result is that the branches in Fig. 4(a) become smoothly connected: they are all part of the same universe.

Unlike when the $\mathbb{Z}_{p}^{(0)}$ symmetry is exact, now there are finite tension domain wall configurations connecting discrete chiral vacua. On the domain wall $\eta$ interpolates between 0 and $2 \pi$ while $\varphi$ interpolates between $2 \pi / p$ and 0 . As an example, the trajectory for $p=3$ connecting the $(\varphi, \eta)=(2 \pi, 0) \rightarrow(4 \pi / 3,2 \pi) \sim(4 \pi / 3,0)$ vacua is shown in Fig. 6. The domain wall connecting neighboring chiral vacua follows a straight trajectory in field space parameterized by $\varphi(\tau)=\frac{2 \pi k}{p}-\alpha(\tau), \eta=p \alpha(\tau)$. In terms of $\alpha$, the domain wall is simply a Sine-Gordon kink

$$
\alpha(\tau)=\frac{4}{p} \arctan \left[\exp \left(\sqrt{\frac{4 \pi p c}{1+p^{2}} M \tau}\right)\right],
$$

with tension $T \sim M$ up to $\mathcal{O}(1)$ factors.

As we increase $M$ the height of the barrier between vacua increases in the smooth direction in field space, while the height of the barrier in the cuspy direction where only $\varphi$ varies stays fixed. When $M$ is large, even though the domain wall passing through the large barrier seems more costly than the domain wall passing through the cusp, the

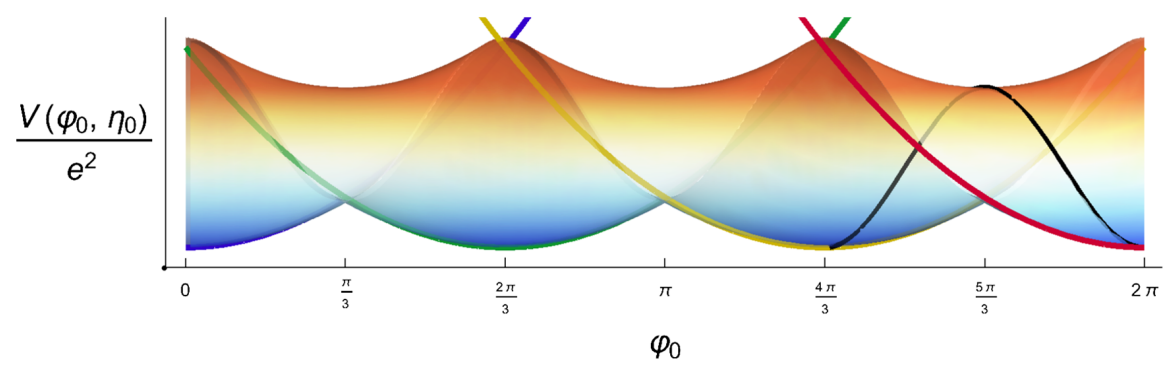

FIG. 6. Domain wall configuration between two chiral symmetry breaking vacua for $p=3, \theta=0$. Rather than pass through the cusp, the domain wall trajectory must go above the barrier, whose height scales as $M^{2}$. The curves coincide with the four branches in Fig. 4(a). 


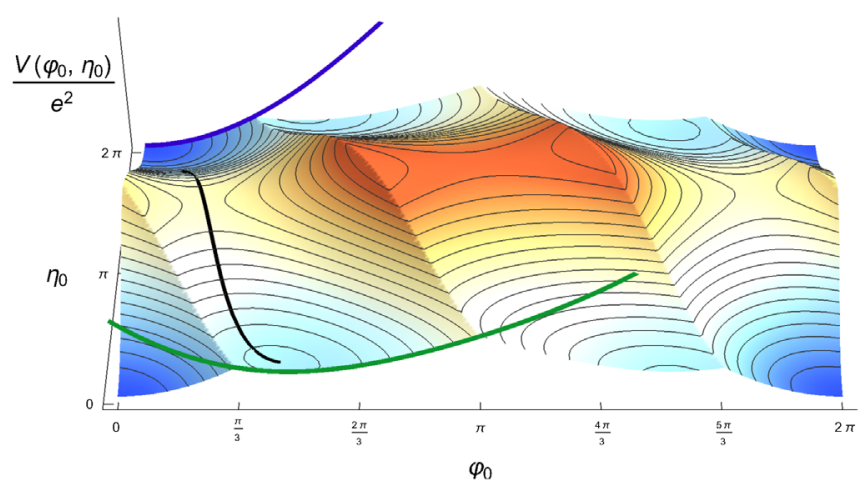

FIG. 7. Effective potential for $p=3, \theta=0, m>0$, and $M>0$. The bounce solution renders the false vacuum unstable. The blue and green curves coincide with the branches in Fig. 4(b).

latter configuration is still not admissible. When $M \rightarrow \infty$ there are no finite-tension domain walls. Indeed, in the formal limit $M \rightarrow \infty$ the unit charge particle decouples and we land on the charge- $p$ model which has $p$ universes.

If we also introduce a mass $m$ for the charge- $p$ fermion the chiral symmetry is broken completely. When $\theta=0$ there is a single unique vacuum at $\varphi=\eta=0$, as illustrated in Fig. 7. Unlike the theory without charge-1 fields [see Fig. 4(b)], the metastable vacua can decay via the nucleation of bubbles of true vacuum. Despite the fact that the false vacuum has an energy density scaling with $m$ relative to the true vacuum, the decay rate can be made arbitrarily small by increasing $M$. The decay rate of the false vacuum scales as $\sim e^{-S_{I}}$ where $S_{I}$ is the action of a bounce solution, which has the form

$$
S_{I}=-\pi R^{2} \varepsilon+2 \pi R T,
$$

where $\varepsilon \sim m \mu$ is the difference in energy density of the two minima and $T$ is the tension of the bounce solution, which to leading order in a large $M$ expansion is given by the domain wall tension $T \sim M$. The size of the bubble of true vacuum is $R=T / \epsilon$, so the action of the bounce configuration $S_{I}=\pi T^{2} / \epsilon$ scales as $M^{2} /(m \mu)$ for $M$ large. When $m$ is small compared to $|e|, \mu \sim e$. Hence, the lifetime of the false vacuum is exponentially enhanced by the ratio of a 'UV' scale $M$ and the low-energy scales $e$ and $m$,

$$
\tau \sim e^{S_{I}} \sim e^{M^{2} /(e m)},
$$

leading to near-eternal false vacua when $M^{2} \gg \mathrm{em}$.

\section{B. Charge-1 Schwinger model with a modified instanton sum}

So far we have discussed the massless and massive charge- $p$ Schwinger models, and their extensions to include charge- 1 fermions. Here we provide a different construction that leads to the same physics. As pointed out in Refs. [23,24], restricting the instanton number in the $2 d$
$\mathbb{C} \mathbb{P}^{N-1}$ model to be a multiple of $p$ gives rise to the same physics as the charge- $p \mathbb{C} \mathbb{P}^{N-1}$ model. Indeed, this correspondence applies to all $U(1)$ gauge theories in two dimensions. For $d>2$ the relation between modified instanton sums and nonminimal charge assignments breaks down. However, theories with modified instanton sums share the features of $2 \mathrm{~d}$ Abelian theories with charge- $p$ matter, namely the existence of universes and false vacua. To set the stage for our treatment of $4 d$ theories with modified instanton sums, we explain the $2 \mathrm{~d}$ correspondence in detail.

Consider a generic $2 \mathrm{~d} U(1)$ gauge theory with a charge-1 fermion (a nearly identical discussion holds for bosonic matter),

$\mathcal{L}=\frac{1}{2 e^{2}}|d a|^{2}+\bar{\psi}\left(\gamma^{\mu} \partial_{\mu}+i a\right) \psi+\frac{i \theta}{2 \pi} d a+\cdots$,

where the ellipses denote other terms allowed by gauge invariance (we do not impose chiral symmetry for the moment). We now modify the theory so that only topological charges which are multiples of $p$ contribute to the partition function [24]. To this end, let us introduce another $U(1)$ gauge field $\hat{a}$ and a $2 \pi$-periodic compact scalar Lagrange multiplier $\chi$, so that the Lagrangian is shifted by

$$
\delta \mathcal{L}=\frac{i}{2 \pi} \chi \wedge(d a-p d \hat{a})+\frac{i \hat{\theta}}{2 \pi} d \hat{a}
$$

where $\hat{\theta}$ is a new $2 \pi$-periodic theta parameter. Integrating out $\chi$ sets $d a=p d \hat{a}$, which means

$$
\frac{1}{2 \pi} \int d a=\frac{p}{2 \pi} \int d \hat{a} \in p \mathbb{Z}
$$

so that only topological charges (for $a$ ) which are multiples of $p$ contribute to the path integral. As a result, $\theta$ has a reduced periodicity, $\theta \sim \theta+2 \pi / p$.

On the other hand, the equation of motion for $\hat{a}$ sets $\chi=$ constant, while integrating out $\hat{a}$ and performing the sum over its topological sectors sets $\chi=2 \pi k / p$ for $k=0, \ldots, p-1$. The path integral sums over $\ell$, so that

$$
Z_{\text {constrained }}(\theta)=\sum_{k=0}^{p-1} Z_{\text {original }}(\theta+2 \pi k / p),
$$

which makes manifest the $2 \pi / p$-periodicity of $\theta$.

To see that the charge- 1 theory with a modified instanton sum is equivalent to the corresponding charge- $p$ theory with a standard instanton sum, we simply solve the local constraint imposed by $\chi$. Substituting $d a=p d a \hat{~ i n t o ~ t h e ~}$ Lagrangian,

$\mathcal{L}=\frac{p^{2}}{2 e^{2}}|d \hat{a}|^{2}+\bar{\psi}\left(\gamma^{\mu} \partial_{\mu}+i p \hat{a}\right) \psi+\frac{i(p \theta+\hat{\theta})}{2 \pi} d \hat{a}$. 
If we now rescale the gauge coupling $e=p \hat{e}$, and define $\tilde{\theta}=p \theta+\hat{\theta}$, this is just a charge- $p U(1)$ gauge theory with $\theta$ parameter $\tilde{\theta}$ and no modification to the instanton sum. The gauge coupling rescaling is harmless-the only role of $e$ is to set the overall energy scale for the theory.

All of the discussions above are about universes in $2 d$ gauge Abelian gauge theory coupled to charge- $p$ matter applies verbatim to the corresponding theories with minimally-charged matter and modified instanton sums. Uplifting the former construction to higher dimensions only gives rise to a $\mathbb{Z}_{p} 1$-form symmetry, while the latter gives rise to the $\mathbb{Z}_{p}(d-1)$-form symmetry necessary for the existence of universes and eternal false vacua.

To make the connection to the charge- $p$ model more explicit, it is worth discussing the 0-form chiral symmetry of the charge-1 Schwinger model with a modified instanton sector, whose bosonized form is

$$
\begin{aligned}
\mathcal{L}= & \frac{1}{2 e^{2}}|d a|^{2}+\frac{1}{8 \pi}|d \varphi|^{2}+\frac{i}{2 \pi} \varphi \wedge d a \\
& +\frac{i}{2 \pi} \chi \wedge(d a-p d \hat{a}) .
\end{aligned}
$$

Using the arguments in the previous paragraph, this theory is equivalent to the charge- $p$ Schwinger model. One might ask what, in the modified instanton sum description, is the analog of the discrete chiral symmetry $\mathbb{Z}_{p}^{(0)}$ of the charge- $p$ Schwinger model. The fact that $e^{i \chi}$ becomes, after integrating out $\hat{a}$, a discrete field valued in the $p$ th roots of unity leads to a mild violation of cluster decomposition also observed in the context of Gerby conformal field theories $[23,25]$. Relatedly, the symmetry transformation properties of $e^{i \chi}$ are subtle and should be treated carefully.

In the bosonized variables, a first guess might be to define a shift symmetry which takes $\varphi \rightarrow \varphi+2 \pi / p$ and does not act on $\chi$. For reference, let us compute the path integral over $\hat{a}$ and $\chi$,

$$
\begin{aligned}
& \int \mathcal{D} \hat{a} \mathcal{D} \chi e^{-\frac{i}{2 \pi} \int \varphi \wedge d a-\frac{i}{2 \pi} \int \chi \wedge(d a-p d \hat{a})} \\
& =\int \mathcal{D} \hat{f} \mathcal{D} \chi \sum_{k \in \mathbb{Z}} e^{i \int\left(k+\frac{p}{2 \pi} \chi\right) \hat{f}} e^{-\frac{i}{2 \pi} \int \varphi \wedge d a-\frac{i}{2 \pi} \int \chi \wedge d a} \\
& =\frac{2 \pi}{p} \sum_{k \in \mathbb{Z}} \int \mathcal{D} \chi \delta\left(\chi+\frac{2 \pi k}{p}\right) e^{-\frac{i}{2 \pi} \int \varphi \wedge d a-\frac{i}{2 \pi} \int \chi \wedge d a} \\
& =\frac{2 \pi}{p} \sum_{k \in \mathbb{Z}} e^{\frac{i k}{p} \int d a} e^{-\frac{i}{2 \pi} \int \varphi \wedge d a} \\
& =2 \pi \sum_{m \in \mathbb{Z}} \delta\left(\frac{1}{2 \pi} \int d a-p m\right) e^{-\frac{i}{2 \pi} \int \varphi \wedge d a}
\end{aligned}
$$

where in the first equality we defined $\hat{f}=d \hat{a}$. As expected, the TQFT sector in (3.23) simply enforces a constraint on the topological charge of the gauge field $a$.

The shift $\varphi \rightarrow \varphi+2 \pi / p$ does not leave the exponentiated action $e^{-S}$ invariant. However, it does leave the partition function invariant, since

$$
\begin{aligned}
& \int \mathcal{D} \hat{a} \mathcal{D} \chi e^{-\frac{i}{2 \pi} \int \varphi \wedge d a-\frac{i}{2 \pi} \int \chi \wedge(d a-p d c)} \\
& \rightarrow 2 \pi \sum_{m \in \mathbb{Z}} \delta\left(\frac{1}{2 \pi} \int d a-p m\right) e^{-\frac{i}{2 \pi} \int \varphi \wedge d a} e^{-\frac{i}{p} \int d a}
\end{aligned}
$$

This is equivalent to (3.27) because the delta function setting $\int d a \in 2 \pi p \mathbb{Z}$ ensures that the extra phase $e^{-\frac{i}{p} \int d a}$ is trivial. The invariance of the path integral is only manifest after integrating out both $\hat{a}$ and $\chi$. Now let us calculate the expectation values of $\left\langle e^{i \varphi}\right\rangle$ and $\left\langle e^{i \chi}\right\rangle$. Again, just from the path integral over $\hat{a}$ and $\chi$, we have

$$
\begin{aligned}
& \int \mathcal{D} \hat{a} \mathcal{D} \chi e^{i \varphi} e^{-\frac{i}{2 \pi} \int \varphi \wedge d a-\frac{i}{2 \pi} \int \chi \wedge(d a-p d \hat{a})} \\
& =2 \pi \sum_{m \in \mathbb{Z}} \delta\left(\frac{1}{2 \pi} \int d a-p m\right) e^{i \varphi} e^{-\frac{i}{2 \pi} \int \varphi \wedge d a}
\end{aligned}
$$

From this it is clear that under the shift $\varphi \rightarrow \varphi+2 \pi / p$, we have

$$
\left\langle e^{i \varphi}\right\rangle \rightarrow e^{2 \pi i / p}\left\langle e^{i \varphi}\right\rangle
$$

On the other hand, the expectation value $\left\langle e^{i x}\right\rangle$ naively does not transform at all, since at the level of the fundamental fields the symmetry acts only on $\varphi$. But in fact it turns out that $\left\langle e^{i \chi}\right\rangle \rightarrow e^{-2 \pi i / p}\left\langle e^{i \chi}\right\rangle$ when $\varphi \rightarrow \varphi+2 \pi / p$. To see this note that the relevant part of the expectation value is

$$
\begin{aligned}
& \int \mathcal{D} \hat{a} \mathcal{D} \chi e^{i \chi} e^{-\frac{i}{2 \pi} \int \varphi \wedge d a-\frac{i}{2 \pi} \int \chi \wedge(d a-p d \hat{a})} \\
& =2 \pi \sum_{m \in \mathbb{Z}} \delta\left(\frac{1}{2 \pi} \int d a-1-p m\right) e^{-\frac{i}{2 \pi} \int \varphi \wedge d a} .
\end{aligned}
$$

We see that in the presence of the insertion $e^{i \chi}$ the constraint on the topological sectors of $a$ is modified. As a result, when we shift $\varphi \rightarrow \varphi+2 \pi / p$, the extra phase $e^{-\frac{i}{p} \int d a}$ is a nontrivial root of unity, and

$$
\left\langle e^{i x}\right\rangle \rightarrow e^{-2 \pi i / p}\left\langle e^{i x}\right\rangle
$$

From the point of view of our (naive) definition of the symmetry action on fields, this is peculiar: we did not assign the field $\chi$ a transformation under the symmetry, yet correlation functions behave as if $\chi$ were charged. 


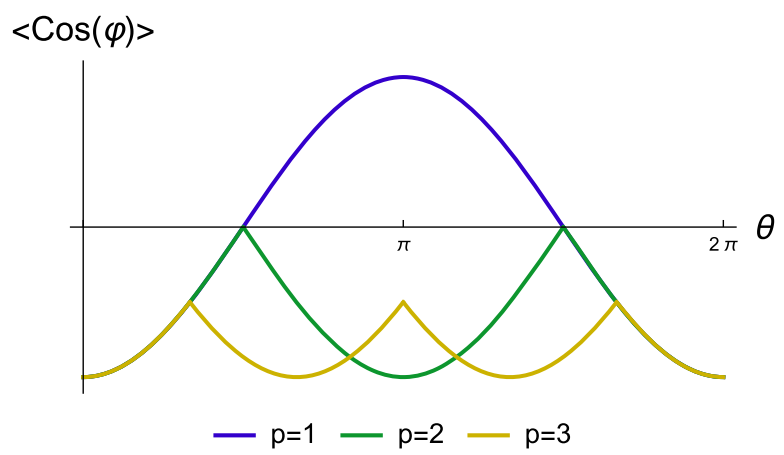

FIG. 8. The $\theta$-dependence of the chiral condensate $\bar{\psi} \psi \sim \cos \varphi$ in the Schwinger model with a very small positive fermion mass $0<m \ll e$ and a modified instanton sum characterized by an integer $p$. The unmodified theory has $p=1$.

The action of chiral symmetry on the expectation values of $\varphi$ and $\chi$ found in (3.30) and (3.32) is consistent with the identification of $U_{k}[C]=e^{-i k \int_{C}\left(\hat{a}-\frac{i}{2 p} \star d \varphi\right)}$ as the charge operators of the $\mathbb{Z}_{p}^{(0)}$ symmetry. One can easily check that $\left\langle U_{k}[C] e^{i \varphi(x)}\right\rangle=e^{2 \pi i k / p}\left\langle e^{i \varphi(x)}\right\rangle$ and $\left\langle U_{k}[C] e^{i \chi(x)}\right\rangle=$ $e^{-2 \pi i k / p}\left\langle e^{i \chi(x)}\right\rangle$ when the closed curve $C$ has linking number 1 with the point $x$. However, there are no sensible charge operators that act as $\left\langle\tilde{U}[C] e^{i \varphi(x)}\right\rangle=e^{2 \pi i / p}\left\langle e^{i \varphi(x)}\right\rangle$ and $\left\langle\tilde{U}[C] e^{i \chi(x)}\right\rangle=\left\langle e^{i \chi(x)}\right\rangle$. For instance, the natural candidates for such operators, $\tilde{U}_{k}[C]=e^{-i k \int_{C}\left(\frac{a}{p}-\frac{i}{2 p} \star d \varphi\right)}$, are not gauge-invariant.

The upshot of this discussion is that to make the action of the chiral symmetry on fields have the simplest correspondence to its action in correlation functions, we should define the action of $\mathbb{Z}_{p}^{(0)}$ as

$$
\mathbb{Z}_{p}^{(0)}: \varphi \rightarrow \varphi+\frac{2 \pi}{p}, \quad \chi \rightarrow \chi-\frac{2 \pi}{p} .
$$

With this definition, one can verify that the exponentiated action $e^{-S}$ itself is left invariant thanks to flux quantization condition for $\hat{a}$ alone, without integrating out $\chi$ explicitly. This implies that the expectation values of both $e^{i \varphi}$ and $e^{i \chi}$ transform with the expected phases. Passing back to the fermionic formulation of the theory, we see that the chiral symmetry transformation becomes

$$
\mathbb{Z}_{2 p}^{(0)}: \psi \rightarrow e^{i \frac{2 \pi}{2 p} \gamma} \psi, \quad \chi \rightarrow \chi-\frac{2 \pi}{p} .
$$

Finally, let us give an example of how the $\theta$-dependence of local observables becomes $2 \pi / p$-periodic in accordance with the discussion near Eq. (3.20). Consider the expectation value $\langle\cos \varphi\rangle$ which is proportional to $\langle\bar{\psi} \psi\rangle$ in the fermionic variables. Setting $\hat{\theta}=0$ without loss of generality, the expectation value can be computed in the bosonic variables by introducing a source $J \cos (\varphi)$, minimizing the free energy with respect to $\varphi$, and taking a derivative with respect to $J$ before sending $J \rightarrow 0$. As $\theta$ increases from 0 , the value of $\varphi$ minimizing the free energy varies continuously until $\theta$ crosses $\pi / p$, where it jumps discontinuously as the minimal-energy universe changes from $k=0$ to $k=1$. This happens again each time $\theta$ crosses a multiple of $\pi / p$, resulting in the cuspy $\theta$-dependence of the chiral condensate shown in Fig. 8.

\section{UNIVERSES AND ETERNAL FALSE VACUA IN 4d}

Our goal in this section is to discuss eternal false vacua in $4 \mathrm{~d}$ gauge theories. Most of this section is a direct generalization of our discussion in two dimensions. Our focus will be on $S U(N)$ QCD with $N_{f}$ fundamental fermions and a modified instanton sum, which (when the fermions are massive) features eternal false vacua for generic values of parameters. This was already noted in [8] for Yang-Mills theory and one-flavor QCD, and our discussion in the opening of this section will mostly be a summary of the ideas of $[8,24]$.

It is useful to first warm up by recalling the fate of topological charge in QCD coupled to an axion $\chi$. The $2 \pi$ periodic axion scalar field couples to QCD's gauge fields through the term

$$
\frac{i}{8 \pi^{2}} \int_{M_{4}} \chi \wedge \operatorname{tr} F \wedge F
$$

where $F$ is the $S U(N)$ 2-form field strength and $M_{4}$ is a closed Euclidean spacetime manifold. Let $\chi_{0}$ denote the zero mode of $\chi$. Suppose that the $U(1)_{\mathrm{PQ}}$ PecceiQuinn symmetry shifting $\chi \rightarrow \chi+c$ is only broken by the coupling to QCD. ${ }^{6}$ Then QCD instantons generate a potential for the effective theta parameter $\theta_{\text {eff }}=\chi_{0}+\theta$, which is minimized when $\theta_{\text {eff }}=0$, rendering the theory $C P$ invariant [28-35]. In this scenario the zero mode of $\chi$ only enters linearly in the action, and we can integrate $\chi_{0}$ out explicitly, yielding

$$
\int d \chi_{0} e^{-i \chi_{0} \int_{M_{4}} \frac{1}{8 \pi^{2}} \operatorname{tr}[F \wedge F]}=2 \pi \delta_{Q_{T}, 0}
$$

where $Q_{T}=\int_{M_{4}} \frac{1}{8 \pi^{2}} \operatorname{tr}[F \wedge F]$ is the topological charge. We see that integration over the zero mode of the QCD axion implements a constraint that gauge configurations with non-vanishing topological charge do not contribute to the partition function. The only way for instantons to contribute to the partition function is through the effects of instanton-antiinstanton pairs.

\footnotetext{
${ }^{6}$ This is a strong version of the "axion quality" assumption which refers to the constraint that $U(1)_{\mathrm{PQ}}$-breaking effects in the UV do not spoil $\theta_{\text {eff }} \approx 0[26,27]$.
} 
The above global constraint on the QCD partition function arose because we introduced a new propagating degree of freedom, namely the axion. Can one constrain the instanton sum without introducing any new propagating degrees of freedom? The answer is yes $[8,24]$. The following action (with a slight abuse of notation) suffices:

$$
\begin{aligned}
S= & \int_{M_{4}}\left[\frac{1}{2 g^{2}} \operatorname{tr}[F \wedge \star F]+\frac{i \theta}{8 \pi^{2}} \operatorname{tr}[F \wedge F]\right. \\
& \left.+\sum_{i=1}^{N_{f}}\left(\bar{\psi}_{i} \gamma^{\mu}\left(\partial_{\mu}-i a_{\mu}\right) \psi_{i}+m \bar{\psi}_{i} \psi_{i}\right)\right] \\
& +\int_{M_{4}}\left[i \chi \wedge\left(\frac{1}{8 \pi^{2}} \operatorname{tr} F \wedge F-\frac{p}{2 \pi} d c^{(3)}\right)+\frac{i \hat{\theta}}{2 \pi} d c^{(3)}\right],
\end{aligned}
$$

where we take $p$ to be a positive integer. The top line is the standard action for QCD on the spacetime $M_{4}: F=$ $d a+i a \wedge a$ where $a$ is the $S U(N)$ gauge field, $\psi_{i}$ are Dirac fermion fields in the fundamental representation of the $S U(N)$ gauge group, with the conventional $\theta$ and mass parameter $m$. The lower line restricts the instanton sum by introducing a 3 -form $U(1)$ gauge field $c^{(3)}$ and $2 \pi$ periodic scalar Lagrange multiplier $\chi^{7}$. The theory enjoys 0 -form $S U(N)$ gauge invariance as well as a 2-form gauge invariance $c^{(3)} \rightarrow c^{(3)}+d \lambda^{(2)}$ where $\lambda^{(2)}$ is a 2-form $U(1)$ gauge function, meaning that $\int d \lambda^{(2)} \in 2 \pi \mathbb{Z}$ on closed 3 -manifolds. This implies the flux quantization condition

$$
\frac{1}{2 \pi} \int_{M_{4}} d c^{(3)} \in \mathbb{Z}
$$

where $M_{4}$ is any closed Euclidean four-manifold, so that $\hat{\theta}$ is a $2 \pi$-periodic parameter. The $\mathbb{Z}_{N}^{(1)}$ center symmetry of pure $S U(N)$ YM theory is explicitly broken by the fundamental-representation fermions.

The fields $c^{(3)}$ and $\chi$ with the coupling given in (4.3) do not carry any propagating degrees of freedom, and hence do not introduce any new dynamics. ${ }^{8}$ The degrees of freedom

\footnotetext{
${ }^{7}$ We have explicitly included the associated topological angle $\hat{\theta}$ for the topological charge density $\frac{d c^{(3)}}{2 \pi}$ in addition to the usual QCD $\theta$ parameter. The fact that one can perform field redefinitions of $\chi$ ensures that only the linear combination $p \theta+\hat{\theta}$ can be observable. As usual, when at least one quark flavor is massless $p \theta+\hat{\theta}$ can be eliminated by a chiral rotation, but when the quarks are massive the value of $p \theta+\hat{\theta}$ is measurable mod $2 \pi$.

${ }^{8}$ This assertion relies on the fact that we did not write kinetic terms for $\chi$ and $c^{(3)}$. Kinetic terms for 3 -form gauge fields are technically irrelevant in four spacetime dimensions: the 3-form gauge coupling has mass dimension 2 . This makes it consistent to exclude a kinetic term for $c^{(3)}$ in (4.3). But to the extent one can neglect such a kinetic term, the $c^{(3)}$ equation of motion sets $d \chi=0$, so it is also consistent to exclude a $\chi$ kinetic term.
}

described by (4.3) are precisely the same as standard QCD. To see that introducing these fields restricts the instanton sum, we first note that the equation of motion for $\chi$ is

$$
\frac{1}{8 \pi^{2}} \operatorname{tr} F \wedge F=\frac{p}{2 \pi} d c^{(3)} .
$$

Equating the integrals of both sides over closed fourmanifolds implies that $S U(N)$ instantons can only contribute to the partition function if their topological charge is divisible by $p$. As a corollary, this immediately implies that the $\theta$ periodicity is $2 \pi / p$. At the same time, the equation of motion of $c^{(3)}$ is

$$
d \chi=0
$$

This means that integrating out $c^{(3)}$ sets $\chi$ to be constant, and recalling the global constraint that $\int d c^{(3)} \in 2 \pi \mathbb{Z}$ leads to the condition $\chi=2 \pi k / p$, where $k=0,1, \ldots p-1$. This means that $e^{i \chi(x)}$ is a topological local operator (i.e., independent of $x$ ), with expectation values $\left\langle e^{i \chi}\right\rangle=e^{2 \pi i k / p}$. The path integral over $\chi$ then sums over $k$, so that

$$
\begin{aligned}
Z_{\text {constrained }}(\theta, \hat{\theta}) & =\sum_{k=0}^{p-1} Z_{\text {original }}(\theta+2 \pi k / p, \hat{\theta}-2 \pi k) \\
& =\sum_{k=0}^{p-1} Z_{\text {original }}(\theta+2 \pi k / p, \hat{\theta})
\end{aligned}
$$

where we used the fact that $\hat{\theta}$ is $2 \pi$ periodic. This expression, which is identical to the $2 \mathrm{~d}$ expression (3.21), gives another way of seeing that $\theta$ is $2 \pi / p$ periodic. It also shows that the partition function decomposes into universes labeled by $k$, or equivalently, the expectation values $\left\langle e^{i x}\right\rangle \in \mathbb{Z}_{p}$.

\section{A. Discrete symmetries and mixed anomaly}

Let us now investigate the discrete global symmetries of the theory more closely. We will discuss the continuous symmetries and their interplay with the discrete symmetries in the next section.

Let us start with the 0 -form internal discrete symmetries. In contrast to standard massless QCD, when $m=0$ there is an enlarged 0 -form discrete chiral symmetry $\mathbb{Z}_{2 N_{f} p}^{(0)}$ due to the modified instanton sum. As discussed in Sec. III B, consistency with the expected transformations of correlation functions requires us to define the $\mathbb{Z}_{2 N_{f} p}^{(0)}$ symmetry as a combination of chiral rotations of the fermions and shifts of the Lagrange multiplier field $\chi$. Consider the $\mathbb{Z}_{2 N_{f} p}$ transformations generated by

$$
\mathbb{Z}_{2 N_{f} p}^{(0)}: \psi_{i} \rightarrow e^{\frac{2 \pi i}{2 N_{f}} \gamma_{5}} \psi_{i}, \quad \chi \rightarrow \chi-\frac{2 \pi}{p}
$$


The chiral rotation shifts the action by

$$
\delta_{\psi} S=\frac{2 \pi i}{2 N_{f} p} \times 2 N_{f} \int_{M_{4}} \frac{1}{8 \pi^{2}} \operatorname{tr} F \wedge F
$$

while the shift of $\chi$ induces a shift of the action by

$$
\delta_{\chi} S=-\frac{2 \pi i}{p} \int_{M_{4}}\left[\frac{1}{8 \pi^{2}} \operatorname{tr} F \wedge F-\frac{p}{2 \pi} d c^{(3)}\right],
$$

and the invariance of the path integral under any chiral rotation generated by (4.8) follows from the fact that $\int_{M_{4}} d c^{(3)} \in 2 \pi \mathbb{Z}$. The $\mathbb{Z}_{2}$ subgroup of $\mathbb{Z}_{2 N_{f} p}^{(0)}$ is fermion parity, while the $\mathbb{Z}_{N_{f}}$ subgroup lies within $S U\left(N_{f}\right)_{A}$. Thanks to the modified instanton sum, the discrete part of the chiral symmetry group is an extension of $\mathbb{Z}_{2 N_{f}}$ by $\mathbb{Z}_{p}$ described by the short exact sequence

$$
1 \rightarrow \mathbb{Z}_{2 N_{f}} \rightarrow \mathbb{Z}_{2 N_{f} p} \rightarrow \mathbb{Z}_{p} \rightarrow 1 .
$$

The standard 't Hooft interaction term $\operatorname{det} \bar{\psi}_{i} \psi_{j}$ is only invariant under $\mathbb{Z}_{2 N_{f}} \subset \mathbb{Z}_{2 N_{f} p}^{(0)}$, so it cannot be radiatively generated when $m=0$. But $e^{i \chi} \operatorname{det} \bar{\psi}_{i} \psi_{j}$ is invariant under $\mathbb{Z}_{2 N_{f} p}^{(0)}$, so it can be radiatively generated at $m=0$. In any given universe, $\chi$ is a constant, so $e^{i \chi} \operatorname{det} \bar{\psi}_{i} \psi_{j}$ looks just like the standard 't Hooft interaction term. This makes $\mathbb{Z}_{2 N_{f} p}^{(0)}$ a rather peculiar chiral symmetry: in any given universe, it does not forbid the generation of operators that look asymmetric. But $\mathbb{Z}_{2 N_{f} p}^{(0)}$ does constrain the form these operators take in the whole collection of universes, in such a way that the QFT as a whole is invariant.

A nonzero common fermion mass $m$ breaks $S U\left(N_{f}\right)_{A}$ completely and breaks the discrete chiral symmetry down to fermion number, $\mathbb{Z}_{2 N_{f} p}^{(0)} \rightarrow \mathbb{Z}_{2}$. When $m \neq 0$ there remains a vectorlike faithfully acting $U\left(N_{f}\right) / \mathbb{Z}_{N}$ zero-form global symmetry, which will play an important role below.

QCD with a modified instanton sum also has a 3-form global symmetry $\mathbb{Z}_{p}^{(3)}$. One way to see that this must be the case is to observe that the $c^{(3)}$ gauge field couples with charge $p$ to the (tautologically) conserved current $\star d \chi$. If $\chi$ were a propagating axion field, $\star d \chi$ would be the conserved current for the 2-form $U(1)$ "axion string" global symmetry. Gauging an $n$-form $U(1)$ global symmetry with charge $p$ always leads to a $(n+1)$-form $\mathbb{Z}_{p}$ global symmetry, as is familiar from QED coupled to matter with charge $p$. Indeed, in modified QCD, the topological operator $e^{i \chi}$ can be interpreted as the symmetry operator for a 3-form $\mathbb{Z}_{p}^{(3)}$ symmetry acting on three-dimensional charged objects, which can be viewed as Wilson surfaces $V\left[M_{3}\right]=\exp \left[i \int_{M_{3}} c^{(3)}\right]$. The existence of the global symmetry is encoded in the topological correlation function

$$
\left\langle e^{i \chi(x)} V\left[M_{3}\right]\right\rangle=e^{\frac{2 \pi i}{p} \operatorname{Link}\left(x, M_{3}\right)}\left\langle V\left[M_{3}\right]\right\rangle,
$$

where $\operatorname{Link}\left(x, M_{3}\right)$ is the linking number of the point $x$ and the closed 3-manifold $M_{3}$. The 3-form global symmetry can also be described in terms of a transformation acting on the fields: it takes $c^{(3)} \rightarrow c^{(3)}+\lambda^{(3)}$, where $\lambda^{(3)}$ is locally a closed, but not exact, 3-form, and globally satisfies $\int_{M_{3}} \lambda^{(3)} \in 2 \pi \mathbb{Z} / p$.

The fact that the symmetry operator of $\mathbb{Z}_{p}^{(3)}$, namely $e^{i \chi}$, is itself charged under $\mathbb{Z}_{2 N_{f} p}^{(0)}$ implies that there is a mixed anomaly between these two symmetries. To see this explicitly we turn on background gauge fields for the 3 -form symmetry. This amounts to introducing a pair of 3 -form and 4-form background gauge fields $D^{(3)}, D^{(4)}$, and requiring that under a background 3 -form gauge transformation $c^{(3)} \rightarrow c^{(3)}+\Lambda^{(3)}$ these background gauge fields transform as

$D^{(3)} \rightarrow D^{(3)}+p \Lambda^{(3)} \quad D^{(4)} \rightarrow D^{(4)}+d \Lambda^{(3)}$.

We assume that $D^{(3)}$ is a properly normalized $U(1)$ gauge field, so that $\int_{M_{4}} d D^{(3)} \in 2 \pi \mathbb{Z}$. The fields $D_{3}$ and $D_{4}$ couple to $c^{(3)}$ via minimal coupling, $p c^{(3)} \rightarrow p c^{(3)}-D^{(3)}$, and $d c^{(3)} \rightarrow d c^{(3)}-D^{(4)}$. To make sure that the $D$ fields describe a $\mathbb{Z}_{p}$ higher-form gauge theory, we take the $D$ fields to have a BF-type action:

$$
\frac{i}{2 \pi} \int_{M_{4}} \tilde{\chi} \wedge\left(p D^{(4)}-d D^{(3)}\right)+\frac{i h}{2 \pi} \int_{M_{4}} D^{(4)}
$$

where $\tilde{\chi}$ is a scalar Lagrange multiplier, and $h=$ $0,1, \ldots, p-1$ is a discrete theta parameter. The equation of motion for $\tilde{\chi}$ ensures that $\int_{M_{4}} D^{(4)} \in \frac{2 \pi}{p} \mathbb{Z}$. At the same time, the equation of motion for $\chi$ now becomes

$$
\frac{1}{8 \pi^{2}} \operatorname{tr} F(a) \wedge F(a)=\frac{1}{2 \pi}\left(p d c^{(3)}-d D^{(3)}\right) .
$$

Applying a chiral transformation shifts the action by

$$
\delta S=i \int_{M_{4}}\left(d c^{(3)}-D^{(4)}\right) .
$$

We therefore find the following $\mathbb{Z}_{2 N_{f} p}^{(0)}$ transformation of the path integral in the presence of background fields for $\mathbb{Z}_{p}^{(3)}$ :

$Z\left(\theta, \hat{\theta}, h ; D^{(3)}, D^{(4)}\right) \stackrel{\mathbb{Z}_{2 N_{f} p}}{\longrightarrow} e^{-i \int D^{(4)}} Z\left(\theta, \hat{\theta}, h ; D^{(3)}, D^{(4)}\right)$

Since $\int D^{(4)} \in \frac{2 \pi}{p} \mathbb{Z}$, the phase on the right-hand side is nontrivial and cannot be eliminated by any choice of local 
counter-terms. Thus QCD with a modified instanton sum has a $\mathbb{Z}_{p}$ 't Hooft anomaly associated with the quotient $\mathbb{Z}_{p} \simeq \mathbb{Z}_{2 N_{f} p}^{(0)} / \mathbb{Z}_{2 N_{f}}$ and the $\mathbb{Z}_{p}^{(3)}$ symmetry. ${ }^{9}$

Standard assumptions about the behavior of QCD when $N_{f}<N_{f}^{*}$, where $N_{f}^{*}$ is the lower edge of the conformal window, imply that when $m=0$, the discrete and continuous chiral symmetries are spontaneously broken on $\mathbb{R}^{4}$ when $N_{f}$ is not too large, with vacua characterized by the phases of order parameters such as $\sum_{i} \bar{\psi}_{i} \psi_{i}$ and $\operatorname{det} \bar{\psi}_{i} \psi_{j} .{ }^{10}$ This means that the Goldstone manifold (which is nontrivial when $N_{f}>1$ ) has $p$ disconnected components corresponding to the $p$ distinct universes. As noted above, it is always possible to write the partition function of QCD with a modified instanton sum as

$$
Z(\theta)=\sum_{k=0}^{p-1} Z(\theta+2 \pi k / p)
$$

When $N_{f}>1$ and the approximate chiral symmetry at small $m$ is spontaneously broken, one can describe the lowenergy dynamics using the chiral field $U=e^{i \Pi / f_{\pi}}$, where $\Pi$ is a Hermitian matrix and $f_{\pi}$ is the Nambu-Goldstone boson decay constant. This must be done separately in each of the terms in (4.18), leading to $p$ disconnected Goldstone manifolds $U_{k}, k=0, \ldots, p-1$.

Finally, we note that the 3 -form symmetry is spontaneously broken so long as the $\mathbb{Z}_{2 N_{f} p}^{(0)}$ is not explicitly broken. The argument showing that $\mathbb{Z}_{p}^{(3)}$ breaks spontaneously is completely analogous to the argument in Sec. III A showing that the $\mathbb{Z}_{p}^{(1)}$ symmetry breaks spontaneously in the charge- $p$ Schwinger model. The insertion of the extended operator $V\left[M_{3}\right]$ in the path integral separates spacetime into two regions. Inside the four-dimensional subregion bounded by $M_{3}$, the insertion of $V\left[M_{3}\right]$ has the same effect as a discrete chiral rotation relative to the outside region. Therefore, as long as $\mathbb{Z}_{2 N_{f} p}^{(0)}$ is not explicitly broken, the energy cost of inserting $V\left[M_{3}\right]$ does not scale with the spacetime volume bounded by $M_{3}$. This is equivalent to saying that the expectation values of the extended operator $V\left[M_{3}\right]$ follow a perimeter law, so the $\mathbb{Z}_{p}^{(3)}$ symmetry is spontaneously broken.

\section{B. Higher-group symmetry}

We now turn to the continuous zero-form symmetries of QCD with a modified instanton sum. It turns out that the 3 -form discrete symmetry and the continuous global

\footnotetext{
${ }^{9}$ It is also possible to interpret (4.17) as an anomaly in the space of couplings (see Refs. [36,37]) for the $\theta$ parameter.

${ }^{10}$ We expect that the mixed 't Hooft anomaly is matched by massless chiral fermions within the conformal window at large $N_{f}$.
}

symmetry of the theory do not form a direct product. Instead, they mix in a nontrivial way, and form a highergroup symmetry structure, see [38] for an introduction to higher groups in QFT. Higher group symmetries in QFT have been studied in a variety of contexts in recent work, see, e.g., [8,39-53]. In particular, Tanizaki and Ünsal [8] showed that pure $4 \mathrm{~d}$ Yang-Mills theory with a modified instanton sum has a 4-group global symmetry due to an intertwining between 1 -form $\mathbb{Z}_{N}$ center symmetry and a $\mathbb{Z}_{p}$ 3-form global symmetry. Here we show that $N_{f} \geq 1$-flavor QCD with a modified instanton sum also has a 4-group global symmetry, this time arising due to an intertwining of a continuous zero-form global symmetry with a $\mathbb{Z}_{p} 3$-form global symmetry. We will show that if one turns on certain background gauge fields for the continuous zero-form symmetries, then one necessarily induces nontrivial fluxes for background gauge fields for the discrete 3-form global symmetry.

Suppose that all of the flavors of quarks have a common mass $m \neq 0$. Then the continuous transformations leaving the action invariant are

$$
\frac{S U(N) \times U\left(N_{f}\right)}{\mathbb{Z}_{N}}
$$

where the $S U(N)$ factor comes from color gauge transformations, the $U\left(N_{f}\right)$ factor comes from flavor rotations, and the quotient involves the $\mathbb{Z}_{N}$ rotations common to both factors. The physical states are in representations of the faithfully-acting symmetry group $U\left(N_{f}\right) / \mathbb{Z}_{N}$. But as observed in Ref. [54], this means that if we turn on an $U\left(N_{f}\right) / \mathbb{Z}_{N}$ flavor background field which is not an $U\left(N_{f}\right)$ gauge field, then the dynamical gauge field would be forced to be in a $P S U(N)=S U(N) / \mathbb{Z}_{N}$ bundle, rather than in an $S U(N)$ bundle.

To illustrate how this works, we use the techniques introduced in [15,55], see also [56-63]. We will turn a $U(1)_{B}^{(0)}=U(1) / \mathbb{Z}_{N}$ baryon number background gauge field, and will find that in general this leads to the dynamical color gauge field living in $S U(N) / \mathbb{Z}_{N}$. One could also probe the theory by turning on a full non-Abelian $U\left(N_{f}\right) / \mathbb{Z}_{N}$ flavor background, but for our purposes it is sufficient to focus on Abelian flavor backgrounds.

Let us first describe $S U(N) / \mathbb{Z}_{N}$ gauge fields. One way to do this is to couple $S U(N)$ gauge fields to a classical $\mathbb{Z}_{N}$ TQFT. The fields of the TQFT are the one-form and twoform background gauge fields $B^{(2)}, B^{(1)}$, with an action

$$
S_{\mathrm{TQFT}}=\frac{i}{2 \pi} \int_{M_{4}} \varphi^{(2)} \wedge\left(N B^{(2)}-d B^{(1)}\right)
$$

where $\varphi^{(2)}$ is a 2 -form Lagrange multiplier. The TQFT is invariant under $U(1)$ 1-form background gauge transformations $B^{(2)} \rightarrow B^{(2)}+d \lambda^{(1)}, B^{(1)} \rightarrow B^{(1)}+N \lambda^{(1)}$, with 
$\int_{M_{2}} d \lambda^{(1)} \in 2 \pi \mathbb{Z}$ on closed 2-manifolds. Note that the flux of $B^{(2)}$ on any closed manifold $M_{2}$ is fractional, $\int_{M_{2}} B^{(2)} \in \frac{2 \pi}{N} \mathbb{Z}$. To couple this TQFT to our gauge theory, we replace the $S U(N)$ gauge field $a$ by the $U(N)$ gauge field $\tilde{a}$, which we assign the 1 -form gauge transformation $\tilde{a} \rightarrow \tilde{a}+\lambda^{(1)}$, and introduce the extra term in the action

$$
S_{U(N) \text { constraint }}=\frac{i}{2 \pi} \int_{M_{4}} \eta^{(2)} \wedge\left(\operatorname{tr} \tilde{F}-N B^{(2)}\right)
$$

where $\eta$ is another 2-form Lagrange multiplier and $\tilde{F}$ is the $U(N)$ field strength. The $U(N)$ gauge field coupled to $B^{(1)}, B^{(2)}$ in the manner given above is a way to write an $S U(N) / \mathbb{Z}_{N}$ gauge field. To write the $S U(N) / \mathbb{Z}_{N}$ gauge field part of the action, we replace

$$
\int_{M_{4}}\left[\frac{1}{2 g^{2}} \operatorname{tr} F \wedge \star F+\frac{i \theta}{8 \pi^{2}} \operatorname{tr} F \wedge F\right]
$$

by

$$
\begin{aligned}
& \int_{M_{4}}\left[\frac{1}{2 g^{2}} \operatorname{tr}\left(\tilde{F}-B^{(2)} \mathbf{1}\right) \wedge \star\left(\tilde{F}-B^{(2)} \mathbf{1}\right)\right. \\
& \left.+\frac{i \theta}{8 \pi^{2}} \operatorname{tr}\left(\tilde{F}-B^{(2)} \mathbf{1}\right) \wedge\left(\tilde{F}-B^{(2)} \mathbf{1}\right)\right]
\end{aligned}
$$

in the Lagrangian.

We now review the fact that the color gauge field in QCD lies in $S U(N) / \mathbb{Z}_{N}$ if we simultaneously turn on a generic background gauge field for $U(1)_{B}^{(0)}$. A $U(1)_{B}^{(0)}$ gauge field can be described as a quark number gauge field $A$ coupled to a $\mathbb{Z}_{N}$ TQFT built out of gauge fields $C^{(1)}, C^{(2)}$ with the constraint $N C^{(2)}=d C^{(1)}$. The flux of $C^{(2)}$ is fractional, $\int_{M_{2}} C^{(2)} \in \frac{2 \pi}{N} \mathbb{Z}$. The fields $A, C^{(1)}, C^{(2)}$ transform under 1-form gauge transformations as $A \rightarrow A+\tilde{\lambda}^{(1)}$, $C^{(1)} \rightarrow C^{(1)}+N \tilde{\lambda}^{(1)}, C^{(2)} \rightarrow C^{(2)}+d \tilde{\lambda}^{(1)}$. All this implies that the quark part of the action of our original $S U(N)$ gauge theory should be replaced by

$$
\int_{M_{4}} \bar{\psi} \gamma^{\mu}\left[\partial_{\mu}-i\left(\tilde{a}-\frac{B^{(1)}}{N}\right)-i\left(A-\frac{C^{(1)}}{N}\right)\right] \psi .
$$

The $1 / N$ charges of $\psi$ under the $B^{(1)}$ and $C^{(1)}$ gauge fields mean that this expression would be inconsistent with charge quantization if only one of $B^{(1)}$ or $C^{(1)}$ are activated. But there is no inconsistency as long as both $C^{(1)}$ and $B^{(1)}$ are turned on, with fluxes that obey $\int_{M_{2}} B^{(2)}+\int_{M_{2}} C^{(2)} \in 2 \pi \mathbb{Z}$.

Now, in the presence of a nontrivial $U(1)_{B}^{(0)}$ background, consider the term in the action responsible for implementing the constraint on instanton number. Since the dynamical gauge field is in $\operatorname{PSU}(N)$, we should replace $F$ with the
$U(N)$ field strength $\tilde{F}$, with $\operatorname{tr} \tilde{F}=N B^{(2)}$ as above. The Lagrange multiplier term becomes

$$
\frac{i}{2 \pi} \int_{M_{4}}\left[\chi \wedge\left(\frac{1}{4 \pi} \operatorname{tr} \tilde{F} \wedge \tilde{F}-p d c^{(3)}\right)\right] .
$$

To make this invariant under background 1-form gauge transformations parametrized by $\lambda^{(1)}$, it is tempting to further replace $\tilde{F} \rightarrow \tilde{F}-B^{(2)} \mathbf{1}$. However, the result is only gauge invariant up to boundary terms. To make the action completely gauge invariant, we must also turn on a background gauge field for the $\mathbb{Z}_{p}^{(3)}$ symmetry. As described in the preceding section, this involves turning on higher-form gauge fields $D^{(4)}, D^{(3)}$, leading to

$$
\frac{i}{2 \pi} \int_{M_{4}}\left[\chi \wedge\left(\frac{1}{4 \pi} \operatorname{tr} \tilde{F} \wedge \tilde{F}-p d c^{(3)}+d D^{(3)}\right)\right] .
$$

This expression is invariant under 1-form gauge transformations if $D^{(3)}$ transforms as

$D^{(3)} \rightarrow D^{(3)}-\left(\frac{N}{2 \pi} B^{(2)} \wedge \lambda^{(1)}+\frac{N}{4 \pi} \lambda^{(1)} \wedge d \lambda^{(1)}\right)$.

But then we must also replace (4.14) with the 1-form gauge-invariant expression

$$
\begin{aligned}
& \frac{i}{2 \pi} \int_{M_{4}} \tilde{\chi} \wedge\left(p D^{(4)}-d D^{(3)}-\frac{N}{4 \pi} B^{(2)} \wedge B^{(2)}\right) \\
& \quad+\frac{i h}{2 \pi} \int_{M_{4}} D^{(4)} .
\end{aligned}
$$

As a result we get the relation

$$
p D^{(4)}=d D^{(3)}+\frac{N}{4 \pi} B^{(2)} \wedge B^{(2)}
$$

just as in the pure-Yang-Mills case analyzed in [8], so that in general $\int D^{(4)} \in \frac{2 \pi}{N p} \mathbb{Z}$. Equation (4.29) signals that the theory has a higher-group symmetry: the 0-form and 3 -form symmetries do not form a direct product. Instead, turning on appropriate background gauge fields for the 0-form $U(1)_{B}^{(0)}$ global symmetry also requires one to turn on background fields for the 3-form $\mathbb{Z}_{p}^{(3)}$ global symmetry. ${ }^{11}$

What is the realization of the 4-group symmetry at long distances? As with any other symmetry structure, highergroup symmetry can constrain renormalization group (RG)

\footnotetext{
${ }^{11}$ When $p=1$, the 3 -form global symmetry becomes trivial, and the combination $A^{(3)}=c^{(3)}-D^{(3)}$ defines an ordinary gauge field for the $U(1)^{(2)}$ 2-form axion string symmetry with field strength $G^{(4)}=d c^{(3)}-D^{(4)}$. Provided $c^{(3)}$ is interpreted as a nondynamical background gauge field, the higher-group structure (4.29) then corresponds to the 3-group found in [51].
} 
flows and symmetry-realization patterns. This has been studied in detail for 2-groups built from continuous 0-form and 1-form symmetries by Cordova, Dumitrescu, and Intriligator [38]. One of the results of Ref. [38] is that the 2-group structure implies that it is inconsistent for the continuous 1-form symmetry to be spontaneously broken if the continuous 0 -form symmetry is not spontaneously broken. Reference [38] also discussed constraints from 2-group symmetry if the symmetries involved in the 2-group are emergent in the infrared. In addition, Ref. [38] was also able to place bounds on the ratio of energy scales at which these symmetries emerge, while Ref. [45] discussed analogous constraints for some systems with 3-group symmetries. The heuristic idea behind the observations of Ref. [38] is that the zero-form symmetry participating in a 2-group is not a good subgroup of the 2-group: turning on generic fluxes for background gauge fields of the zero-form symmetry induces fluxes for the background gauge fields of the 1-form symmetry. But fluxes for 1-form symmetry background fields do not induce fluxes for 0 -form symmetry background fields, so the 1-form symmetry counts as a 'good subgroup' of the 2-group symmetry. These observations motivate the conclusion proved explicitly in Ref. [38] that it is only consistent for the 2-group symmetries discussed in Ref. [38] to spontaneously break either to nothing or to the higher-form symmetry group. The 2-groups discussed in Ref. [38] cannot spontaneously break to the 0 -form symmetry group.

The heuristic picture we summarized above also applies for the 4-group structure discussed in this paper. The $U\left(N_{f}\right) / \mathbb{Z}_{N}$ symmetry group is not a good subgroup of the 4-group symmetry of QCD with a constrained instanton sum in the sense that fractional background fluxes for $U\left(N_{f}\right) / \mathbb{Z}_{N}$ induce nontrivial fluxes for background gauge fields for the $\mathbb{Z}_{p}^{(3)}$ symmetry. This observation may make it tempting to guess that the results of Ref. [38] apply to the 4group symmetry discussed here, which would imply that our 4-group symmetry cannot be spontaneously broken to $U\left(N_{f}\right) / \mathbb{Z}_{N}$.

However, a naive generalization along these lines does not work. From the discussion at the end of Sec. IV A we know that $\mathbb{Z}_{p}^{(3)}$ is spontaneously broken at $m=0$, while for $m>0$ it is not spontaneously broken. When $m \gg \Lambda$, the $U(1)_{B}^{(0)}$ symmetry will not be spontaneously broken, and it turns out that the same is true at $m=0$. To show this, suppose that $\mathcal{O}_{f}$ is an order parameter for $U\left(N_{f}\right) / \mathbb{Z}_{N}$. If $m=0$ we find

$$
\begin{aligned}
\left\langle\mathcal{O}_{f}\right\rangle & =\frac{\sum_{k=0}^{p-1} \int \mathcal{D}[\text { fields }] e^{-S\left[\text { fields } ; \theta=\frac{2 \pi k}{p}\right]} \mathcal{O}_{f}}{\sum_{k=0}^{p-1} Z\left(\theta=\frac{2 \pi k}{p}\right)} \\
& =\frac{\sum_{k=0}^{p-1} Z\left(\theta=\frac{2 \pi k}{p}\right)\left\langle\mathcal{O}_{f}\right\rangle_{\theta=\frac{2 \pi k}{p}}}{\sum_{k=0}^{p-1} Z\left(\theta=\frac{2 \pi k}{p}\right)}=0
\end{aligned}
$$

where the expectation value on the left is evaluated in QCD with a constrained instanton sum, while the partition functions and expectation values in the middle are evaluated in QCD with an unconstrained instanton sum. The final equality on the right follows because there is no $\theta$-dependence in the chiral limit $m \rightarrow 0$, so that $\left\langle\mathcal{O}_{f}\right\rangle_{\theta=\frac{2 \pi k}{p}}$ is equal, up to a possible overall phase, to $\left\langle\mathcal{O}_{f}\right\rangle_{\theta=0}$. The VafaWitten theorem [64] then implies that the vectorlike $U\left(N_{f}\right) / \mathbb{Z}_{N}$ cannot be spontaneously broken at $\theta=0$, so that $\left\langle\mathcal{O}_{f}\right\rangle_{\theta=0}$. So $U\left(N_{f}\right) / \mathbb{Z}_{N}$ is not spontaneously broken either at $m=0$ or large $m$, and it is natural to expect that it is not spontaneously broken for any positive $m$. Hence, while there is evidence that the 4-group symmetry is not spontaneously broken at all for $m>0$, when $m=0$ the 4-group symmetry it is clear that it is spontaneously broken to $U\left(N_{f}\right) / \mathbb{Z}_{N}$. This is in contradiction with a naive generalization of the constraints given in Ref. [38]. It would be interesting to do a general study of the constraints of 4-group symmetries on renormalization group flows, particularly when the groups involved are discrete.

\section{Eternal false vacua}

When the $\mathbb{Z}_{p}^{(3)}$ symmetry is exact, the domain walls separating sectors characterized by different phases $\arg \left(\operatorname{det} \bar{\psi}_{i} \psi_{j}\right)$ have infinite tensions, paralleling what we saw in our $2 \mathrm{~d}$ example. In infinite volume the $p$ chiral vacua themselves have an "infinite-fold" degeneracy due to the spontaneous breaking of $S U\left(N_{f}\right)_{A}$. The $p$ sectors do not mix even in finite volume. This implies that even if we increase the temperature of the system enough to restore $S U\left(N_{f}\right)_{A}, p$ degenerate discrete chiral vacua remain. Modified QCD thus features "persistent order" in the sense of [14]. If we add the term $\operatorname{det} \bar{\psi}_{i} \psi_{j}$ to the Lagrangian and explicitly break $\mathbb{Z}_{2 N_{f} p}^{(0)} \rightarrow \mathbb{Z}_{2 N_{f}}$, the $p$ sectors become nondegenerate, and there is a unique minimal-energy ground state. But the nonminimal-energy false vacuum states still cannot decay, and each one can still be called a distinct universe. To decay, bubbles of the true vacuum would have to nucleate and expand through a false vacuum. But in the present context the bubble walls have infinite tension thanks to the unbroken $\mathbb{Z}_{p}^{(3)}$ symmetry. So QCD with a modified instanton sum features eternal false vacua.

The eternal false vacua can be rendered noneternal by coupling QCD with a modified instanton sum to dynamical 2-branes with unit charge under $\mathbb{Z}_{p}^{(3)}$. One way to achieve this is to replace the instanton-number-constraint term in the action

$$
\frac{i}{2 \pi} \int_{M_{4}} \chi \wedge\left(\frac{1}{4 \pi} \operatorname{tr} F \wedge F-p d c^{(3)}\right)
$$

with 


$$
\frac{i}{2 \pi} \int_{M_{4}} \chi \wedge\left(\frac{1}{4 \pi} \operatorname{tr} F \wedge F-\frac{p}{4 \pi} \operatorname{tr} F^{\prime} \wedge F^{\prime}\right)
$$

where $F^{\prime}$ is the field strength for some dynamical $S U\left(N^{\prime}\right)$ gauge field with strong scale $\Lambda^{\prime}$. Integrating out $\chi$ imposes the constraint that only $S U(N)$ instantons with topological charge divisible by $p$ contribute to the path integral. The $p=1$ version of (4.32) was recently considered in Ref. [65] as part of a model to ameliorate the strong $C P$ axion quality problem. Equation (4.32) does not lead to an exact 3-form symmetry for any $p$. Consequently, there is no way to forbid loops of $S U\left(N^{\prime}\right)$ gauge bosons from generating a kinetic term for $\chi$. Moreover, the $\chi \rightarrow \chi+2 \pi / p$ shift symmetry is still present with (4.32), and in the limit $\Lambda^{\prime} \gg \Lambda$ we expect an effective action for the axionlike field $\chi$ of the form

$$
S_{\chi}=\frac{1}{2} \int_{M_{4}}\left[f^{2}|d \chi|^{2}+\mu^{4}(1-\cos (p \chi)+\cdots)\right],
$$

where $f, \mu \sim \Lambda^{\prime}$. The field $\chi$ is now dynamical and has $p$ vacua, and one can think of $\frac{i p}{2 \pi} \int_{M_{4}} \chi \wedge d c^{(3)}$ as the longdistance effective field theory description of these vacua as noted in Ref. [8]. ${ }^{12}$ So there is an emergent 3-form symmetry in the infrared when $\Lambda^{\prime} \gg \Lambda$. The 3-form symmetry is not exact because (4.33) has dynamical domain-wall excitations with tensions $\sim\left(\Lambda^{\prime}\right)^{3}$ which carry charge 1 under $\mathbb{Z}_{p}^{(3)}$.

We can estimate the decay rate per unit volume for the discrete vacua using a standard thin-wall bubble nucleation calculation [5]:

$$
\frac{\Gamma}{V} \sim \mathcal{E} \exp \left[-\frac{27 \pi^{2}}{2} \frac{T_{2}{ }^{4}}{\mathcal{E}^{3}}\right]
$$

where $T_{2}$ is the tension of a unit charge membrane (in the example above $\left.T_{2} \sim\left(\Lambda^{\prime}\right)^{3}\right)$ and $\mathcal{E}$ is the difference in vacuum energy densities between the true and false vacua. If we break the discrete and continuous chiral symmetries by turning on a flavor symmetric soft mass $m, \mathcal{E}$ is set by the physics of QCD and so has a characteristic scale $m \Lambda_{\mathrm{QCD}}^{3}$, while the membrane tension is a free parameter as far as QCD is concerned, and could be, e.g., $T_{2} \sim M_{\text {Planck }}^{3} \cdot{ }^{13}$ If we write $T_{2}=t_{b}^{3} \Lambda_{\mathrm{QCD}}^{3}, \mathcal{E}=\epsilon^{4} \Lambda_{\mathrm{QCD}}^{4}$, then

$$
\frac{\Gamma}{V} \sim \exp \left[-\frac{27 \pi^{2}}{2}\left(\frac{t_{b}}{\epsilon}\right)^{12}\right]
$$

\footnotetext{
${ }^{12}$ See also Ref. [63] for an interesting discussion of how the 't Hooft anomalies of the $F^{\prime}$ gauge theory are matched by domain walls resulting from this kind of effective action.

${ }^{13}$ The estimate in (4.34) is based on a standard flat-space decay rate calculation, so it is valid provided that the typical bubble size $R \sim T_{2} / \mathcal{E}$ is small compared to the inverse Hubble scale $H_{0}^{-1} \sim 10^{42} \mathrm{GeV}^{-1}$. If $\mathcal{E} \sim(1 \mathrm{GeV})^{4}$, then one should not use (4.34) once $T_{2} \gtrsim\left(10^{-5} M_{\text {Planck }}\right)^{3}$ given that $M_{\text {Planck }} \sim 10^{19} \mathrm{GeV}$.
}

so unless one tunes the 2-brane tension parameter $t_{b} \lesssim \epsilon$, the decay rate of the discrete false vacua is guaranteed to be spectacularly slow.

\section{SUMMARY}

In this paper we have studied quantum field theories exhibiting two somewhat exotic attributes, both arising from the existence of a $(d-1)$-form global symmetry. When the symmetry is exact, the path integral decomposes into distinct universes. When the symmetry is approximate, it is possible to have extremely long-lived false vacua whose decay rates are set by high-scale physics controlling the explicit breaking of the higher form symmetry. After a warm up in quantum mechanics, we examined the phenomena above in generalized versions of the Schwinger model and four-dimensional QCD. In both cases the global modifications can be viewed as restricting the sum over instantons to configurations with topological charge divisible by an integer $p$. The topological degrees of freedom which lead to the restriction on the topological charge also give rise to the $(d-1)$-form symmetry responsible for universes and eternal false vacua.

Despite the global modifications, which lead to a change in the $\theta$ periodicity, when $\theta=0$ the theories are locally indistinguishable from their unmodified counterparts-for example, only extended $(d-1)$-dimensional objects can probe their different universes. One key signature of the modification is the existence of false vacua in generic points in parameter space. Unlike familiar examples of false vacua, the differences in energy densities between the false and true vacua can be large, yet the false vacua are eternalthat is, infinitely long lived-provided the $(d-1)$-form symmetry is exact. The decay rates of false vacua are then set by the tensions of $(d-2)$-branes which break the symmetry explicitly.

In both the Schwinger model and QCD with a modified instanton sum, the lifetimes of these false vacua can be made arbitrarily large by dialing the hierarchy between the tensions of the dynamical $(d-2)$-branes and the intrinsic scales of the theory. The fact that one can get trapped in a false QCD vacuum for an extremely long time suggests that the global modification of the instanton sum is difficult to observe through low-energy measurements near $\theta=0$, and should be viewed as an infinite-fold ambiguity in the global structure of QCD, with a number of parallels to the four-fold global ambiguity of the Standard Model discussed in Ref. [66]. The ambiguity in Ref. [66] arises because of four possible distinct quotients that can be imposed on the Standard Model gauge group, three of which can lead to fractionalized topological charges for the $S U(3), S U(2)$ and $U(1)$ gauge fields. But it is also possible restrict the topological charges for each factor of the gauge group individually (or collectively) to be multiples of some integer(s) with no change to the local dynamics, as explored in this paper for QCD. We will explore the 
phenomenological implications of restrictions on the instanton sum of the Standard Model in future work.

\section{ACKNOWLEDGMENTS}

We are grateful to Z. Komargodski, M. Ünsal, and Y. Tanizaki for discussions that inspired this paper. We also thank M. Shifman and A. Vainshtein for lively and clarifying discussions about the Schwinger model, and are very grateful to P. Draper for extensive discussions on the topics in this work and the suggestion to work out a quantum-mechanical example. A. C. is supported by start-up funds from the University of Minnesota. T. J. is supported by a UMN CSE Fellowship.
[1] I. Kobzarev, L. Okun, and M. Voloshin, Bubbles in metastable vacuum, Sov. J. Nucl. Phys. 20, 644 (1975).

[2] T. D. Lee and G. C. Wick, Vacuum stability and vacuum excitation in a spin-0 field theory, Phys. Rev. D 9, 2291 (1974).

[3] M. Stone, Lifetime and decay of "excited vacuum" states of a field theory associated with nonabsolute minima of its effective potential, Phys. Rev. D 14, 3568 (1976).

[4] M. Stone, Semiclassical methods for unstable states, Phys. Lett. 67B, 186 (1977).

[5] S. R. Coleman, The fate of the false vacuum. 1. Semiclassical theory, Phys. Rev. D 15, 2929 (1977); Erratum Phys. Rev. D 16, 1248 (1977).

[6] J. Callan, G. Curtis, and S. R. Coleman, The fate of the false vacuum. 2. First quantum corrections, Phys. Rev. D 16, 1762 (1977).

[7] Z. Komargodski (private communication).

[8] Y. Tanizaki and M. Ünsal, Modified instanton sum in QCD and higher-groups, J. High Energy Phys. 03 (2020) 123.

[9] Z. Komargodski, K. Ohmori, K. Roumpedakis, and S. Seifnashri, Symmetries and strings of adjoint $\mathrm{QCD}_{2}$, arXiv:2008.07567.

[10] E. Palti, The Swampland: Introduction and review, Fortschr. Phys. 67, 1900037 (2019).

[11] D. Gaiotto, A. Kapustin, Z. Komargodski, and N. Seiberg, Theta, time reversal, and temperature, J. High Energy Phys. 05 (2017) 091.

[12] Y. Kikuchi and Y. Tanizaki, Global inconsistency, 't Hooft anomaly, and level crossing in quantum mechanics, Prog. Theor. Exp. Phys. 2017, 113B05 (2017).

[13] K. Aitken, A. Cherman, and M. Ünsal, Dihedral symmetry in $S U(N)$ Yang-Mills theory, Phys. Rev. D 100, 085004 (2019).

[14] Z. Komargodski, A. Sharon, R. Thorngren, and X. Zhou, Comments on Abelian Higgs models and persistent order, SciPost Phys. 6, 003 (2019).

[15] D. Gaiotto, A. Kapustin, N. Seiberg, and B. Willett, Generalized global symmetries, J. High Energy Phys. 02 (2015) 172.

[16] M. M. Anber and E. Poppitz, Anomaly matching, (axial) Schwinger models, and high-T super Yang-Mills domain walls, J. High Energy Phys. 09 (2018) 076.

[17] M. M. Anber and E. Poppitz, Domain walls in high-T SU(N) super Yang-Mills theory and QCD(adj), J. High Energy Phys. 05 (2019) 151.
[18] T. Misumi, Y. Tanizaki, and M. Ünsal, Fractional $\theta$ angle, 't Hooft anomaly, and quantum instantons in charge- $q$ multi-flavor Schwinger model, J. High Energy Phys. 07 (2019) 018.

[19] A. Armoni and S. Sugimoto, Vacuum structure of charge $\mathrm{k}$ two-dimensional QED and dynamics of an anti D-string near an O1-plane, J. High Energy Phys. 03 (2019) 175.

[20] A. Lawrence, $\theta$-angle monodromy in two dimensions, Phys. Rev. D 85, 105029 (2012).

[21] S. Coleman, Quantum Sine-Gordon equation as the massive Thirring model, Phys. Rev. D 11, 2088 (1975).

[22] S. R. Coleman, More about the massive Schwinger model, Ann. Phys. (N.Y.) 101, 239 (1976).

[23] T. Pantev and E. Sharpe, Notes on gauging noneffective group actions, arXiv:hep-th/0502027.

[24] N. Seiberg, Modifying the sum over topological sectors and constraints on supergravity, J. High Energy Phys. 07 (2010) 070.

[25] S. Hellerman, A. Henriques, T. Pantev, E. Sharpe, and M. Ando, Cluster decomposition, T-duality, and gerby CFT's, Adv. Theor. Math. Phys. 11, 751 (2007).

[26] M. Kamionkowski and J. March-Russell, Planck scale physics and the Peccei-Quinn mechanism, Phys. Lett. B 282, 137 (1992).

[27] S. M. Barr and D. Seckel, Planck-scale corrections to axion models, Phys. Rev. D 46, 539 (1992).

[28] R. D. Peccei and H. R. Quinn, CP Conservation in the Presence of Pseudoparticles, Phys. Rev. Lett. 38, 1440 (1977).

[29] R. D. Peccei and H. R. Quinn, Constraints imposed by CP conservation in the presence of pseudoparticles, Phys. Rev. D 16, 1791 (1977).

[30] S. Weinberg, A New Light Boson?, Phys. Rev. Lett. 40, 223 (1978).

[31] F. Wilczek, Problem of Strong $p$ and $t$ Invariance in the Presence of Instantons, Phys. Rev. Lett. 40, 279 (1978).

[32] A. Zhitnitsky, On possible suppression of the axion hadron interactions. (In Russian), Sov. J. Nucl. Phys. 31, 260 (1980).

[33] J. E. Kim, Weak Interaction Singlet and Strong $C P$ Invariance, Phys. Rev. Lett. 43, 103 (1979).

[34] M. Dine, W. Fischler, and M. Srednicki, A simple solution to the strong $C P$ problem with a harmless axion, Phys. Lett. 104B, 199 (1981). 
[35] M. A. Shifman, A. Vainshtein, and V. I. Zakharov, Can confinement ensure natural $C P$ invariance of strong interactions?, Nucl. Phys. B166, 493 (1980).

[36] C. Córdova, D. S. Freed, H. T. Lam, and N. Seiberg, Anomalies in the space of coupling constants and their dynamical applications I, SciPost Phys. 8, 001 (2020).

[37] C. Córdova, D. S. Freed, H. T. Lam, and N. Seiberg, Anomalies in the space of coupling constants and their dynamical applications II, SciPost Phys. 8, 002 (2020).

[38] C. Córdova, T. T. Dumitrescu, and K. Intriligator, Exploring 2-group global symmetries, J. High Energy Phys. 02 (2019) 184.

[39] J. C. Baez and A. D. Lauda, Higher-dimensional algebra V: 2-Groups, arXiv:math/0307200.

[40] J. Baez and U. Schreiber, Higher gauge theory: 2-Connections on 2-bundles, arXiv:hep-th/0412325.

[41] A. Kapustin and R. Thorngren, Higher symmetry and gapped phases of gauge theories, arXiv:1309.4721.

[42] E. Sharpe, Notes on generalized global symmetries in QFT, Fortschr. Phys. 63, 659 (2015).

[43] Y. Tachikawa, On gauging finite subgroups, SciPost Phys. 8, 015 (2020).

[44] F. Benini, C. Cordova, and P.-S. Hsin, On 2-group global symmetries and their anomalies, J. High Energy Phys. 03 (2019) 118.

[45] T. D. Brennan and C. Cordova, Axions, higher-groups, and emergent symmetry, arXiv:2011.09600.

[46] T. Brauner, Field theories with higher-group symmetry from composite currents, J. High Energy Phys. 04 (2021) 045.

[47] X.-G. Wen, Emergent anomalous higher symmetries from topological order and from dynamical electromagnetic field in condensed matter systems, Phys. Rev. B 99, 205139 (2019).

[48] Z. Wan and J. Wang, Higher anomalies, higher symmetries, and cobordisms I: Classification of higher-symmetry-protected topological states and their boundary fermionic/ bosonic anomalies via a generalized cobordism theory, Ann. Math. Sci. Appl. 4, 107 (2019).

[49] Z. Wan, J. Wang, and Y. Zheng, Higher anomalies, higher symmetries, and cobordisms II: Lorentz symmetry extension and enriched bosonic/fermionic quantum gauge theory, Ann. Math. Sci. Appl. 5, 171 (2020).
[50] Y. Hidaka, M. Nitta, and R. Yokokura, Higher-form symmetries and 3-group in axion electrodynamics, Phys. Lett. B 808, 135672 (2020).

[51] C. Cordova, T. T. Dumitrescu, and K. Intriligator, 2-Group global symmetries and anomalies in six-dimensional quantum field theories, arXiv:2009.00138.

[52] Y. Hidaka, M. Nitta, and R. Yokokura, Global 3-group symmetry and 't Hooft anomalies in axion electrodynamics, J. High Energy Phys. 01 (2021) 173.

[53] N. Iqbal and N. Poovuttikul, 2-Group global symmetries, hydrodynamics and holography, arXiv:2010.00320.

[54] D. Gaiotto, Z. Komargodski, and N. Seiberg, Time-reversal breaking in $\mathrm{QCD}_{4}$, walls, and dualities in $2+1$ dimensions, J. High Energy Phys. 01 (2018) 110.

[55] A. Kapustin and N. Seiberg, Coupling a QFT to a TQFT and duality, J. High Energy Phys. 04 (2014) 001.

[56] H. Shimizu and K. Yonekura, Anomaly constraints on deconfinement and chiral phase transition, Phys. Rev. D 97, 105011 (2018).

[57] A. Cherman, S. Sen, M. Unsal, M. L. Wagman, and L. G. Yaffe, Order Parameters and Color-Flavor Center Symmetry in QCD, Phys. Rev. Lett. 119, 222001 (2017).

[58] Y. Tanizaki and Y. Kikuchi, Vacuum structure of bifundamental gauge theories at finite topological angles, J. High Energy Phys. 06 (2017) 102.

[59] Y. Tanizaki, Anomaly constraint on massless QCD and the role of Skyrmions in chiral symmetry breaking, J. High Energy Phys. 08 (2018) 171.

[60] M. M. Anber and E. Poppitz, On the baryon-color-flavor (BCF) anomaly in vector-like theories, J. High Energy Phys. 11 (2019) 063.

[61] T. Kanazawa and M. Ünsal, Quantum distillation in QCD, Phys. Rev. D 102, 034013 (2020).

[62] M. Ünsal, Strongly coupled QFT dynamics via TQFT coupling, arXiv:2007.03880.

[63] M. M. Anber and E. Poppitz, Deconfinement on axion domain walls, J. High Energy Phys. 03 (2020) 124.

[64] C. Vafa and E. Witten, Restrictions on symmetry breaking in vector-like gauge theories, Nucl. Phys. B234, 173 (1984).

[65] A. Hook, S. Kumar, Z. Liu, and R. Sundrum, High Quality QCD Axion and the LHC, Phys. Rev. Lett. 124, 221801 (2020).

[66] D. Tong, Line operators in the Standard Model, J. High Energy Phys. 07 (2017) 104. 